Published in NeuroImage, Volume 30, Issue 3, 15 April 2006, Pages 813-826.

The original publication is available at www.sciencedirect.com via the following DOI: http://dx.doi.org/10.1016/j.neuroimage.2005.10.014

\title{
Influence of Tissue Conductivity Anisotropy on EEG/MEG Field and Return Current Computation in a Realistic Head Model: A Simulation and Visualization Study using High-Resolution Finite Element Modeling.
}

\author{
C.H. Wolters ${ }^{\mathrm{a}, \mathrm{b}, *}$, A. Anwander ${ }^{\mathrm{c}}$, X. Tricoche ${ }^{\mathrm{b}}$, D. Weinstein ${ }^{\mathrm{b}}$, \\ M.A. Koch ${ }^{\mathrm{d}}$, R.S. MacLeod ${ }^{\mathrm{b}}$ \\ ${ }^{\mathrm{a}}$ Westfälische Wilhelms-Universität Münster, Institut für Biomagnetismus und \\ Biosignalanalyse, Malmedyweg 15, 48149 Münster, Germany \\ ${ }^{\mathrm{b}}$ University of Utah, Scientific Computing and Imaging Institute, 50 S. Central \\ Campus Dr., Room 3493, Salt Lake City, UT 84112, USA \\ ${ }^{\mathrm{c}}$ Max Planck Institute for Human Cognitive and Brain Sciences, Stephanstr. 1a, \\ 04103 Leipzig, Germany \\ ${ }^{\mathrm{d}}$ University Medical Center Hamburg-Eppendorf, Institute for Systems \\ Neuroscience, Martinistr. 52, 20246 Hamburg, Germany
}

\begin{abstract}
To achieve a deeper understanding of the brain, scientists and clinicians use Electroencephalography (EEG) and Magnetoencephalography (MEG) inverse methods to reconstruct sources in the cortical sheet of the human brain. The influence of structural and electrical anisotropy in both the skull and the white matter on the EEG and MEG source reconstruction are not well understood.

In this paper, we report on a study of the sensitivity to tissue anisotropy of the EEG/MEG forward problem for deep and superficial neocortical sources with differing orientation components in an anatomically accurate model of the human head.

The goal of the study was to gain insight into the effect of anisotropy of skull and white matter conductivity through the visualization of field distributions, isopotentialsurfaces, and return current flow and through statistical error measures. One implicit premise of the study is that factors that affect the accuracy of the forward solution will have at least as strong an influence over solutions to the associated inverse problem.
\end{abstract}


Major findings of the study include 1) anisotropic white matter conductivity causes return currents to flow in directions parallel to the white matter fiber tracts; 2) skull anisotropy has a smearing effect on the forward potential computation; and 3) the deeper a source lies and the more it is surrounded by anisotropic tissue, the larger the influence of this anisotropy on the resulting electric and magnetic fields. Therefore, for the EEG, the presence of tissue anisotropy both for the skull and white matter compartment substantially compromises the forward potential computation and as a consequence, the inverse source reconstruction. In contrast, for the MEG, only the anisotropy of the white matter compartment has a significant effect. Finally, return currents with high amplitudes were found in the highly conducting cerebrospinal fluid compartment, underscoring the need for accurate modeling of this space.

Key words: EEG, MEG, source reconstruction, tissue conductivity anisotropy, $\mathrm{CSF}$, forward problem, finite element method, return current, visualization

\section{Introduction}

The inverse problem in EEG and MEG aims at reconstructing the underlying current distribution in the human brain using potential differences and/or magnetic fluxes measured non-invasively directly from the head surface, or from a close distance. The goal of this study was to examine the sensitivity of the associated EEG/MEG forward problem specially to conductive anisotropy within the brain. We computed forward solutions for both isotropic and anisotropic versions of realistic head models using the finite element approach and evaluated the results throughout the head using sophisticated visualization techniques as well as statistical metrics.

A major premise of this study is that there are regions of the head that do not conduct electrical current isotropically, i.e., equally in all directions, but rather they conduct preferentially in directions related to the underlying tissue structure (Geddes and Baker, 1967; Haueisen, 1996). The human skull consists of a soft bone layer (spongiosa) enclosed by two hard bone layers (compacta). Since the spongiosa has a much higher conductivity than the compacta (Akhtari et al., 2002), the skull can be described by an effective anisotropic conductivity with a ratio of up to 1:10 radially to tangentially to the skull surface (Rush and Driscoll, 1968). It is also known that brain white matter has an anisotropic conductivity with a ratio of about 1:10 (normal:parallel to fibers) (Nicholson, 1965), but no direct techniques exist for

\footnotetext{
* Corresponding author. Tel.: +49/251/83-56904, Fax: +49/251/83-56874
}

Email address: $\{$ carsten.wolters\}@uni-muenster.de (C.H. Wolters). 
its robust and non-invasive measurement. Recently, however, formalisms have been described for relating the effective electrical conductivity tensor of brain tissue to the effective water diffusion tensor as measured by Diffusion Tensor Magnetic Resonance Imaging (DT-MRI) (Basser et al., 1994b; Tuch et al., 2001). The underlying assumption is that the same structural features that result in anisotropic mobility of water molecules (detected by DT-MRI) also result in anisotropic conductivity. The quantitative expression for this assumption is that the eigenvectors of the conductivity tensor are the same as those from the water diffusion tensor (Basser et al., 1994b). Even more specifically, Tuch et al. have applied a differential effective medium approach to porous brain tissue and derived a linear relationship between the eigenvalues of the DT and the conductivity tensors (Tuch et al., 2001).

A critical component of source reconstruction is the numerical approximation method used to reach an accurate solution of the associated forward problem, i.e., the simulation of fields for known dipolar sources in the brain. Although there are several different approaches in common use for this type of problem the Finite Element (FE) method is able to treat both realistic geometries and inhomogeneous and anisotropic material parameters (Haueisen, 1996; Buchner et al., 1997; van den Broek et al., 1998; Marin et al., 1998; Schimpf et al., 2002) and so is the approach we employed. Previous work has not sufficiently investigated the impact of tissue anisotropy on EEG and MEG. One impediment to using the FE method - and to this type of modeling in general - has been the high computational cost of carrying out the simulations. The use of recently developed advances in the FE method in EEG/MEG inverse problems (Weinstein et al., 2000; Wolters et al., 2002; Gencer and Acar, 2004; Wolters et al., 2004b) dramatically reduce the complexity of the computations, so that the main disadvantage of FE modeling no longer exists. In realistic FE models, sensitivity studies have been carried out for the influence of skull anisotropy on EEG and MEG (van den Broek et al., 1998; Marin et al., 1998; Wolters, 2003), while, to our knowledge, only a few studies have investigated the influence of realistic white matter anisotropy (Haueisen et al., 2002; Wolters, 2003). Those studies support the hypothesis that modeling anisotropy is crucial for accurate source reconstruction. The major limit of these studies that we have addressed is that their result evaluation was restricted to scalp potentials/fields. In this study we have computed, compared and visualized potentials and especially the return current flow throughout the volume of the head. Those additional informations allow a much more detailed examination of the effects of anisotropy than is possible from the evaluation of scalp values alone.

Using our realistic, anisotropic head model and a variety of sources, we were able to compare throughout the head volume the effects of anisotropic conductivity on bioelectric fields. Our results support those from previous studies suggesting that inclusion of anisotropy can be essential to accurate modeling 
of electric and magnetic fields and, by extension, to accurate source localization. In addition, our results show the nature of the current flow in regions of anisotropy and provide fundamental indications of the interplay between tissue characteristics and bioelectric fields.

\section{Methods}

To carry out the analysis of sensitivity of brain source simulation requires the construction of detailed realistic head models, in this case from MRI image data. Here we outline the steps we used to construct such a model and then apply advanced numerical techniques to the solution of forward problems.

\subsection{MRI data acquisition}

T1-weighted MRI is well suited for the segmentation of tissue boundaries like white and gray matter, outer skull and skin. In contrast, the identification of the inner skull surface is more successful from Proton Density (PD) weighted MRI sequence because the difference in the quantity of water protons between intracranial and bone tissues is large. We regarded the skull and white matter layers as anisotropic compartments, the description of which we obtained from T1-/PD-MRI and whole-head DT-MRI with the associated segmentation process.

\subsubsection{Measurement of T1- and PD-MRI}

MR imaging of a healthy 32 year old male subject was performed on a 3 Tesla whole-body scanner (Medspec 30/100, Bruker, Ettlingen/Germany). For the T1-MRI, an inversion recovery MDEFT sequence (Lee et al., 1995) was employed (flip angle of $25^{\circ}, T R=11.7 \mathrm{~ms}, T E=6 \mathrm{~ms}, T_{M D}=1.3 \mathrm{~s}$ ). For the 3D PD-MRI, acquired one week later, we used a 3D FLASH protocol (Haase et al., 1986) with $T E=6 \mathrm{~ms}$, a flip angle of $25^{\circ}$ and $T R=11.7 \mathrm{~ms}$. The scan resolution was $1 \times 1 \times 1.5 \mathrm{~mm}^{3}$ in both acquisitions, which were linearly interpolated to an isotropic $1 \mathrm{~mm}^{3}$ voxel size.

\subsubsection{Whole head DT-MRI measurements}

Whole-head-DT-MRI was performed using a 4-slice displaced Ultra-Fast Low Angle RARE (U-FLARE) protocol with centric phase-encoding (Norris and Börnert, 1993). Diffusion weighting was implemented as a Stejskal-Tanner type 
spin-echo preparation (Koch, 2000). Although Echo Planar Imaging (EPI) is widely applied for DT-MRI purposes, U-FLARE avoids spatial deformation of the DT-MRI and the resulting misregistration between it and the anatomic $3 \mathrm{D}$ data. The effective echo time was $T E=120 \mathrm{~ms}$, and $T R=11 \mathrm{~s}$. The diffusion weighting gradient pulses had a duration of $\delta=22 \mathrm{~ms}$, and their onset was separated by $\Delta=40 \mathrm{~ms}$. Four different $\mathbf{b}$ matrices with evenly spaced trace $\mathbf{b}$ between 50 and $800 \mathrm{~s} / \mathrm{mm}^{2}$ were applied through variation of the gradient strength (Koch, 2000). The slices were axially oriented and $5 \mathrm{~mm}$ thick with in-plane resolution of $2 \times 2 \mathrm{~mm}^{2}$. In order to increase the signal-to-noise ratio, 5 to 16 images (depending on $\mathbf{b}$ ) with identical diffusion weighting were averaged. Due to the long measurement time (50 min for 4 slices) data acquisition was split into 8 sessions. Diffusion tensor calculation (Basser et al., 1994a) was based on a multivariate regression algorithm in IDL (Interactive Data Language, Research Scientific, Bolder, Colorado/USA). T1-weighted images were acquired in the same session as anatomical reference for the offline registration process.

\subsection{Registration and Segmentation}

To construct a realistic volume conductor model requires segmention of the different tissues within the head with special attention to the poorly conducting human skull and the highly conductive CSF (Hämäläinen and Sarvas, 1987; Cuffin, 1996; Roth et al., 1993; Huiskamp et al., 1999; Ramon et al., 2004).

\subsubsection{T1-/PD-MRI}

In order to correct for different subject positions and geometrical distortions, we first aligned T1- and PD-MRI with a voxel-similarity based affine registration without pre-segmentation using a cost-function based on mutual information (Wolters, 2003). The T1 images provided the information on soft tissues while the registered PD image enabled the segmentation of the inner skull surface.

Our nearly automatic segmentation process consisted of a 3D implementation of an Adaptive Fuzzy C-Means classification method that compensates for image intensity inhomogeneities (based on the original work in two dimensions of Pham and Prince (1999)), followed by a deformable model algorithm to smooth the inner and outer skull surfaces (Wolters, 2003). We segmented five head compartments out of the bimodal dataset; skin, skull, CSF, gray and white matter. In source reconstruction, it is generally accepted that the weak volume currents either outside the skull or far away from EEG and MEG 
sensors have a negligible influence on the measured fields (Bruno et al., 2004). We therefore did not make any effort to segment the face and used instead a cutting procedure like that reported in standard boundary element head modeling (e.g., (Wagner, 1998)).

Please put Figure 1 here.

Figure 1 shows an axial, a coronal and a sagittal cut through a five tissue segmentation result, in which one can observe the segmentation produced by our method.

\subsubsection{DT-MRI}

The coregistered T1 images of the same slices allowed the registration of the DT-MRI data onto the 3D T1 data set. The registered DT data were then resampled to $1 \mathrm{~mm}^{3}$. In order to handle the orientation information in the registered DT images appropriately, the matrix of each diffusion tensor, $\mathbf{D}^{\mathrm{eff}}$, was rotated with the rotation matrix $\mathbf{R}$ of the respective registration process via the similarity transform $\mathbf{D}=\mathbf{R} \mathbf{D}^{\mathrm{eff}} \mathbf{R}^{T}$. Since water diffusion coefficients in CSF are much larger than in the brain, a large contrast was achieved at the brain surface, which provided a quality check of the registration.

Please put Figure 2 here.

Figure 2 shows a map of the Fractional Anisotropy (FA, for the definition see (Basser and Pierpaoli, 1996)) of the registered DT data, masked with the white matter mask from the segmentation procedure. The first row shows the FA values overlaid on the T1-MRI. With $F A=0.74$, the highest value was found in the splenium of the corpus callosum. In the second row, the color coded directions (Pajevic and Pierpaoli, 1999) of the first tensor eigenvector weighted with the FA are presented and overlaid on the T1-MRI. Note the strong anisotropy of the corpus callosum and the pyramidal tracts. Furthermore, as Figure 2 shows, the registered DT-MRI slices were not exactly parallel because the images were acquired in multiple sessions. Any missing values were filled with isotropic tensors with a trace value characteristic of white matter.

\subsection{Volume conductor FE mesh generation}

Please put Figure 3 here.

A prerequisite for $\mathrm{FE}$ modeling is the generation of a mesh that represents the geometric and electric properties of the head volume conductor. To gener- 
ate the mesh, we used the software CURRY (2000) to create a surface-based tetrahedral tessellation of the five segmented compartments. The procedure exploits the Delaunay-criterion, enabling the generation of compact and regular tetrahedra, and is described in detail elsewhere (Wagner, 1998; Wolters, 2003). The process resulted in a finite element model with 147,287 nodes and 892,115 tetrahedra elements as shown in Figure 3.

\subsection{Finite element conductivity}

The finite elements were then labeled according to their compartment membership and assigned the following conductivities for the isotropic reference model (Geddes and Baker, 1967; Rush and Driscoll, 1968; Haueisen, 1996; Baumann et al., 1997): skin $=0.33 \mathrm{~S} / \mathrm{m}$, skull $=0.0042 \mathrm{~S} / \mathrm{m}$ (skull to skin conductivity ratio of approximately $1: 80), \mathrm{CSF}=1.79 \mathrm{~S} / \mathrm{m}$, gray matter $=$ $0.33 \mathrm{~S} / \mathrm{m}$, and white matter $=0.14 \mathrm{~S} / \mathrm{m}$.

\subsubsection{Modeling the skull conductivity anisotropy}

The human skull shows a conductivity with high resistance in the radial direction (as a first approximation, a series connection of a high, a low and a high resistor for inner compacta, spongiosa and outer compacta) and much lower resistance in the tangential directions (parallel connection of the same three resistors) (Rush and Driscoll, 1968).

Determination of the tensor eigenvectors

Marin et al. have pointed out the importance of well-defined skull conductivity tensor eigenvectors by reporting errors in the simulated EEG for the case of an erroneous modeling (Marin et al., 1998). We determined the radial direction from a strongly smoothed triangular mesh, which was shrunken from the outer skull onto the outer spongiosa surface using a discrete deformable surface model (Wolters, 2003).

Please put Figure 4 here.

Figure 4 shows the result on the underlying T1 image. For each skull finite element, we then defined the radial orientation component from the outward normal direction of the computed surface.

\section{Determination of the tensor eigenvalues}

Realistic modeling of the conductivity tensor eigenvalues in the skull is a difficult task, not only because the absolute and relative thicknesses of spongiosa and compacta layers vary and their boundaries are difficult to segment, 
but especially because of inhomogeneous skull resistivity and an inter- and intrasubject variability which can be related to age, diseases, environmental factors, and personal constitution (Rush and Driscoll, 1968; Law, 1993; Haueisen, 1996; Pohlmeier et al., 1997; Ollikainen et al., 1999; Akhtari et al., 2002). We therefore started from the commonly used isotropic conductivity value of $\sigma_{\text {skull }}=0.0042 \mathrm{~S} / \mathrm{m}$ (Huiskamp et al., 1999; Cuffin, 1996; Buchner et al., 1997; Wagner, 1998) and simulated the anisotropic case in the following way: For a given anisotropy ratio, $\sigma^{\text {rad }}: \sigma^{\text {tang }}$, we calculated radial and tangential eigenvalues by obeying one of the following two constraints:

(1) Wang's constraint (Wang et al., 2001), which states that the product of radial and tangential conductivity has to stay constant and has to be equal to the square of the isotropic conductivity:

$$
\sigma^{\mathrm{rad}} \cdot \sigma^{\mathrm{tang}} \stackrel{!}{=} \sigma_{\text {skull }}^{2}
$$

(2) and a volume constraint (Wolters, 2003), which retains the geometric mean of the eigenvalues and thus the volume of the conductivity tensor, i.e.,

$$
\frac{4}{3} \pi \sigma^{\mathrm{rad}}\left(\sigma^{\mathrm{tang}}\right)^{2} \stackrel{!}{=} \frac{4}{3} \pi \sigma_{\text {skull }}^{3}
$$

According to (Rush and Driscoll, 1968; de Munck, 1988; Peters and de Munck, 1991; van den Broek et al., 1998; Marin et al., 1998), the skull has an anisotropy ratio of 1:10. Given the paucity of measurements of skull anisotropy, we decided to include a wide range of values, spanning the only measured value of 1:10 (Rush and Driscoll, 1968) by an order of magnitude in both directions. Our primary goal was then to evaluate the overall effect of anisotropy on the electric and magnetic fields. Table 1 shows the 5 chosen anisotropy ratios and the calculated eigenvalues under the respective constraint.

Please put Figure 5 here.

Figure 5 shows the modeled conductivity tensors of the skull.

\subsubsection{Modeling the white matter conductivity anisotropy}

\section{Determination of the tensor eigenvectors}

Following the proposition of Basser et al. (1994b), we assumed that the conductivity tensors share the eigenvectors with the measured diffusion tensors. Shimony et al. measured diffusion anisotropy in 12 regions of interest in human white and gray matter and showed that in commissural, projection and also association white matter, the shape of the diffusion ellipsoids is strongly prolate ("cigar-shaped"), while gray matter was measured to be close to isotropic (Shimony et al., 1999). Therefore, we assumed prolate rotationally-symmetric 
tensor-ellipsoids for the white matter compartment and modeled the conductivity tensor $\sigma$ for a white matter finite element as

$$
\sigma=\mathbf{S} \operatorname{diag}\left(\sigma^{\text {long }}, \sigma^{\text {trans }}, \sigma^{\text {trans }}\right) \mathbf{S}^{T}
$$

where $\mathbf{S}$ is the orthogonal matrix of unit length eigenvectors of the measured diffusion tensor at the barycenter of the white matter finite element and $\sigma^{\text {long }}$ and $\sigma^{\text {trans }}$ are the eigenvalues parallel (longitudinal) and perpendicular (transverse) to the fiber directions, respectively, with $\sigma^{\text {long }} \geq \sigma^{\text {trans }}$.

\section{Determination of the tensor eigenvalues}

As for the skull compartment, we started from the commonly used isotropic conductivity value of $\sigma_{w m}=0.14 \mathrm{~S} / \mathrm{m}$ for the white matter compartment (Geddes and Baker, 1967; Haueisen, 1996) and used Wang's constraint (see Equation (1)) and the volume constraint (see Equation (2)) to setup the eigenvalues for the anisotropic case. According to (Nicholson, 1965; Tuch et al., 2001; Shimony et al., 1999), the white matter has an anisotropy ratio of 1:10. Given the paucity of direct measurements of white matter conductivity anisotropy (Nicholson, 1965), as for the skull, we decided to include the same wide range of anisotropy ratios also for the white matter compartment (Table 1). Figure 5 presents the normalized and colored (by trace) tensor ellipsoids for 1:2 (volume constraint) skull and white matter anisotropy in the barycenters of the finite elements. Note the left-right and top-bottom anisotropy of the corpus callosum and the pyramidal tract, respectively.

\subsection{Finite element forward modeling}

To represent the relationship between brain sources and bioelectric fields, we made use of the standard approaches to simulation based on the quasistatic Maxwell equations. These lead to an expression of Poisson's equation (Sarvas, 1987)

$$
\nabla \cdot(\sigma \nabla \Phi)=-\nabla \cdot \mathbf{j}^{p} \quad \text { in } \Omega
$$

in which $\mathbf{j}^{p}$ is the primary or impressed current, $\Phi$ is the scalar potential, and $\Omega$ is the head domain. Homogeneous Neumann conditions apply on the head surface $\Gamma=\partial \Omega$,

$$
\left.(\sigma \nabla \Phi \cdot \mathbf{n})\right|_{\Gamma}=0
$$

where $\mathbf{n}$ is the unit surface normal. Additionally, a reference electrode $(\mathrm{FPz})$ is used with given zero potential. For the forward problem, the primary current and the conductivity of the volume conductor are known and the equation is solved for the potential distribution by means of an FE Ansatz. We used a standard variational procedure in order to transform the differential equation (4) into an algebraic system of linear equations (Buchner et al., 1997; Wolters, 
2003). For the modeling of the primary current, we used a "blurred dipole", which has been previously described and intensively validated (Buchner et al., 1997; Wolters, 2003). We solved the resulting high resolution linear equation system, which has a large but sparse symmetric system matrix by means of an iterative Algebraic MultiGrid (AMG) preconditioned conjugate gradient method, which was parallelized for distributed memory computers (Wolters et al., 2002, 2004a). The outstanding performance of the AMG preconditioner in comparison with other methods has been demonstrated previously (Wolters et al., 2000, 2002; Mohr and Vamrunste, 2003). The AMG approach is especially suitable for anisotropic problems and in (Wolters et al., 2002) we showed its stability within this context.

To describe the associated magnetic field from brain sources, one can define

$$
\mathbf{C}(\mathbf{y})=\oint_{\Upsilon} \frac{1}{|\mathbf{x}-\mathbf{y}|} d \mathbf{x},
$$

where $\Upsilon$ is the outer contour of a MEG coil. One can then compute the magnetic flux $\Psi$ at a MEG sensor as (Wolters et al., 2004b):

$$
\begin{aligned}
\Psi & =\Psi_{p}+\Psi_{s}, \text { with } \\
\Psi_{p} & =\frac{\mu}{4 \pi} \int_{\Omega} \mathbf{j}^{p}(\mathbf{y}) \cdot \mathbf{C}(\mathbf{y}) d \mathbf{y}, \text { and } \\
\Psi_{s} & =-\frac{\mu}{4 \pi} \int_{\Omega} \sigma(\mathbf{y}) \nabla \Phi(\mathbf{y}) \cdot \mathbf{C}(\mathbf{y}) d \mathbf{y}
\end{aligned}
$$

In these equations, $\Psi_{p}$ is the so-called primary magnetic flux and $\Psi_{s}$ the secondary magnetic flux, emerging from the primary or the secondary (return) currents, respectively.

To perform these computations, we used the software package NeuroFEM (NeuroFEM, 2000-2005) for EEG and MEG forward modeling. We transformed both the potential distribution within the volume conductor and, independently, the computed distributions at the EEG and MEG sensors to common average reference before error analysis and visualization.

\subsubsection{Simulated sources}

We carried out forward simulation studies for two classes of dipoles, superficial and deep sources. For the class of superficial neocortical sources, we chose two dipoles in the right somatosensory cortex, one of them approximately tangentially oriented (in the posterior-anterior direction) and the other approximately radially oriented (in the inferior-superior direction). Because it is known that both EEG and MEG are especially sensitive to conductivity 
changes in the vicinity of the dipole (Haueisen et al., 2000; Gencer and Acar, 2004), we checked the environment of the superficial somatosensory sources and found that only $15 \%$ of the surrounding finite elements were labeled as white matter and $0 \%$ as skull. The representative of the second class, deep sources, was chosen in the left thalamus, where the source orientation is approximately radial. The thalamus belongs to human gray matter (Shimony et al., 1999), so that the vicinity of the dipole was isotropic. The source strength of each dipole was $100 \mathrm{nAm}$.

\subsection{Simulation setup to assess the influence of anisotropy}

In order to model the EEG, 71 electrodes were placed on the head surface according to the international 10/20 EEG system. For the MEG, we used a BTI ${ }^{1} 148$ channel whole-head system. Each magnetometer flux transformer was modeled by means of a thin, closed conductor loop with a diameter of $11.5 \mathrm{~mm}$, using 8 isoparametric quadratic finite row elements.

We based our evaluation of the effect of anisotropy on forward field modeling on well-known statistical difference metrics, and especially on sophisticated, three-dimensional visualization techniques.

\subsubsection{Statistical difference metrics}

(Meijs et al., 1989) introduced the two difference metrics that we used to compare forward solutions under different conductivity assumptions. The first is the Relative Difference Measure (RDM), defined as

$$
\mathrm{RDM}=\sqrt{\sum_{i=1}^{m}\left(\frac{\underline{\mathrm{u}}_{\mathrm{iSO}}^{[i]}}{\sqrt{\sum_{i=1}^{m}\left(\underline{\mathrm{u}}_{\mathrm{iSO}}^{[i]}\right)^{2}}}-\frac{\underline{\mathrm{u}}_{\mathrm{ani}}^{[i]}}{\sqrt{\sum_{i=1}^{m}\left(\underline{\mathrm{u}}_{\mathrm{ani}}^{[i]}\right)^{2}}}\right)},
$$

where $m$ denotes the number of sensors and $\underline{\underline{i s o}}_{\mathrm{iso}}^{[i]}$ and $\underline{\underline{u}}_{\mathrm{ani}}^{[i]}$ the $i^{\text {th }}$ component of the simulated field vector ( $\underline{u}$ is either the potential $\phi$ or the magnetic flux $\underline{\Psi}$ ) in the isotropic and the anisotropic case, respectively. The RDM is a measure for the topography error (Minimal error: $\mathrm{RDM}=0$ ). The second error measure,

$\overline{1}$ 4-D NeuroImaging, San Diego, USA 
the MAGnification factor (MAG), is defined as

$$
\mathrm{MAG}=\frac{\sqrt{\sum_{i=1}^{m}\left(\underline{\mathrm{u}}_{\mathrm{ani}}^{[i]}\right)^{2}}}{\sqrt{\sum_{i=1}^{m}\left(\underline{\mathrm{u}}_{\mathrm{iso}}^{[i]}\right)^{2}}}
$$

and gives an indication of errors in the magnitude (Minimal error: MAG $=1$ ).

\subsubsection{Visualization of return currents}

In our experience the visualization of return currents $\sigma \mathbf{E}$ is both intuitive and highly informative when trying to understand the effect of anisotropy. Using a Line Integral Convolution (LIC) technique (Cabral and Leedom, 1993), we computed the return current directly over the surface of the head and on coronal slices through the head. This technique permits a continuous depiction of the directional information of the current flow and is combined with a color mapping of the current magnitude that gives insight into the qualitative and quantitative aspects of the current flow.

We also used a technique called stream surfaces (Garth et al., 2004) to assess the influence of tissue conductivity anisotropy. Stream surfaces are defined as surfaces generated by an arbitrary starting curve that is then advected along the vector field. They often constitute a significant improvement over individual streamlines because they provide a better understanding of depth and spatial relationships in the exploration of three-dimensional flows.

\section{Results}

The goal of this study was to evaluate the influence of anisotropic conductivity on the simulation of electric and magnetic fields from dipolar sources in the brain. We present here results from the 3 dipole source types described above and, for each case, compare the results with isotropic and anisotropic assumptions for each of the white matter and the skull. We used a source magnitude of 100nAm and, except for the statistical metrics in Figure 6, we compared the isotropic case with the 1:10 (volume constraint) anisotropic case, which is considered closest to realistic white matter (Nicholson, 1965) and skull anisotropy (Rush and Driscoll, 1968). 


\subsection{Tangentially oriented superficial source}

Please put Figures 6, 7 and 8 here.

Figure 6 (top row) shows the resulting topography (left) and magnification (right) errors for various anisotropy ratios, when either obeying the volume or Wang's constraint. In Figure 7, the EEG and MEG field distribution, linearly interpolated between the sensors (top row), and isopotential-surfaces within the volume conductor (bottom row) are shown for the isotropic case (left), for anisotropy of skull (middle) and white matter compartment (right). In Figure 8, we used the stream surface technique to visualize the effect of skull anisotropy with regard to the return current flow.

Figure 7 and especially 8 clearly show that skull anisotropy smears out and weakens the EEG, resulting in a pattern that looks more like one of a deeper and weaker dipole. In contrast to the isotropic model, the isopotential surfaces for $-5 \mu V$ and $5 \mu V$ were no longer able to break through the skull compartment (Figure 7). Figure 8 furthermore shows the effect of the Neumann boundary conditions (Equation 5) on the return currents, namely that the normal component of the current is zero at the head surface which is expressed by the wide opening of the stream surfaces at the head boundary. Skull anisotropy led to a topography error (RDM) of about $10 \%$ and a magnification factor of about 0.5 (Figure 6, top row, circles). The volume constraint (in black) produced larger errors in comparison to the Wang constraint (in red). Skull anisotropy was found to have no influence $(\mathrm{RDM}<1 \%, \mathrm{MAG} \approx 1)$ on the MEG topography and magnitude for both constraints (not shown in Figure 6).

Including white matter anisotropy (isotropic skull layer) resulted in low RDM $(5 \%)$ and magnitude (MAG of about 0.95 ) errors.

Including anisotropy of both skull and the white matter layer led to a topography error of about $13 \%$ for EEG for both constraints (Figure 6, top row, triangles) which was only marginally higher than the values for skull anisotropy alone.

\subsection{Radially oriented superficial source}

Please put Figure 9 here.

For the case of a radially oriented dipole, Figure 6 (middle row) shows the RDM (left) and MAG (right) errors and Figure 9 the EEG and MEG fields (top row) and isopotential surfaces (bottom row) for the isotropic model (left) and the models with an anisotropic skull compartment (middle) and an anisotropic 
white matter layer (right).

Including anisotropy of the skull (Figure 6, middle row, circles), we found an RDM for the EEG of about $11 \%$ and a MAG of close to 0.5 . Again, the volume constraint (in black) produced slightly bigger errors than Wang's constraint (in red). As Figure 9 shows, skull anisotropy again smeared out and weakened the EEG, the pattern looking like one of a deeper and weaker dipole. In contrast to the isotropic model, the isopotential surfaces for $-1 \mu V$ and $7 \mu V$ were no longer able to break through the skull compartment. As with the tangential superficial source, we found no influence of skull anisotropy on the MEG field distribution.

Including white matter anisotropy had a slightly weaker influence on the topography of the EEG (less than $5 \%$ for both constraints) compared to the tangential dipole case but a larger effect $(\mathrm{MAG}=0.85)$ on the magnitude error (Figure 6, middle row, squares). For the MEG, we note that both RDM and MAG errors are nearly twice as large when compared with the tangential case (Figure 6, middle row, in blue).

If both compartments were simultaneously anisotropic (Figure 6, middle row, triangles), the errors for the EEG were very similar to the errors of pure skull anisotropy, while the errors for the MEG were approximately identical to the errors of white matter anisotropy (not shown).

\subsection{Influence on a deep thalamic source}

Please put Figure 10 here.

Figure 6 (bottom row) shows the resulting RDM and MAG errors and Figure 10 the EEG and MEG fields (top row) and isopotential surfaces (bottom row) for the isotropic model (left) and the models with an anisotropic skull compartment (middle) and an anisotropic white matter layer (right) for the deep thalamic source.

Results in both figures show that for the deep source, with an RDM of more than $10 \%$ for the EEG and more than $15 \%$ for the MEG, white matter anisotropy (Figure 6, bottom row, squares) was the leading cause of topography error. Furthermore, this error was strongly increasing for the 1:100 anisotropy ratio. With a MAG error of about 0.7 , white matter anisotropy strongly weakened the EEG and MEG.

While the topography error was negligible, skull anisotropy (Figure 6, bottom row, circles) strongly weakened the magnitude of the simulated fields, so that the isopotential surfaces for $-3 \mu V$ and $3 \mu V$ in Figure 10 (middle) no longer 
reached the model surface.

Please put Figure 11 here.

Figure 11 shows results from the line integral convolution technique to visualize the return current flow on the surface of the FE model. We found two return current areas of minimal amplitude (in blue), one on the top and one on the bottom of the model (not shown). The amplitude of the return currents was well correlated to the thickness of the skull (compare the color scaling of the return currents with the segmented model in Figure 1). While high return currents were flowing in the thin lateral areas, they were significantly attenuated in the thicker occipital areas and in the areas of the frontal sinuses. The white matter anisotropy mainly weakened the surface return currents.

Please put Figure 12 here.

In Figure 12, we visualized the projection of the return current vector field onto a coronal slice (in black) for the deep thalamic source for the isotropic case and the case of the anisotropic white matter compartment. The amplitude of the return current was color coded on two linear scales, one from 0.3 to 0.003 $A / m^{2}$ in the neighborhood of the source and the second from 0.003 to $0 \mathrm{~A} / \mathrm{m}^{2}$ for remote locations. In the isotropic case, the return currents flowed on nearly circular loops in the classic dipolar pattern. In the anisotropic case, we observe that the main direction component (main eigenvector) of the conductivity tensors, i.e., the main fiber direction, and the computed return current in the white matter compartment are highly parallel.

Please put Figure 13 here.

The results in Figure 13 support this observation by showing the cosine (color coded from 0 to 1 ) of the angle between the main eigenvector of the white matter conductivity tensor in the anisotropic model (its projection onto the coronal plane is shown in black) and the return current vector (not shown here) for a slice in the isotropic model and the model with anisotropic white matter compartment. While in the isotropic case, values close to 1 appeared just by chance, in the anisotropic case, there was close concordance between current direction and local fiber orientation, as the areas of red and yellow coloring in Figure 13 show. The white matter anisotropy thus strongly influenced the flow of the return currents and therefore the EEG and MEG.

Please put Figure 14 here.

In Figure 14, we applied the LIC visualization technique to the return currents on a coronal slice of the model color coded with the return current amplitude for the isotropic (top row) and anisotropic (bottom row) white matter com- 
partments to further quantify the effect of volume conduction for the deep source. Our first observation was that the currents close to the source and, because of its high conductivity, in the CSF compartment, have relatively high amplitudes. With regard to the white matter compartment, the figure further underscores our hypotheses of increased return current flow along the fiber bundles in the anisotropic model (bottom row) when compared to the isotropic case (top row). This figure also shows the effect of the poorly-conducting skull compartment; current flowed along the inner skull boundary, entered the skull, and penetrated it in a clearly radial direction while its amplitude was strongly weakened; it entered the skin compartment and fulfilled the Neumann condition at the head surface, i.e., the condition that the normal component of the current is zero, by either flowing tangentially to the surface or having a zero amplitude on top and on the bottom of the model (compare to the areas with zero amplitude in Fig. 11).

\section{Discussion and Conclusion}

In this paper, we built a realistic finite element head volume conductor model taking into account skull and white matter anisotropy. We exploited a combined T1-/PD-MRI dataset for the construction of a five-tissue model with an anisotropic skull compartment and a whole-head DT-MRI dataset to determine white-matter anisotropy. Our goal was to study the influence of anisotropic tissue conductivity on forward EEG and MEG computations. We used sophisticated high resolution visualization techniques and statistical error quantifications to provide insights into the effect of anisotropy.

For a superficial tangentially oriented source in the somatosensory cortex, our results concerning the influence of skull anisotropy on the EEG potential distribution are in agreement with the observations of others (Marin et al., 1998; van den Broek et al., 1998). We visualized the effect of skull anisotropy on the return currents and showed that skull anisotropy smears out and weakens the EEG, resulting in a pattern that looks more like that of a deeper and weaker dipole.

The MEG results, in contrast, suggest that skull anisotropy has no influence $(\mathrm{RDM}<1 \%, \mathrm{MAG} \approx 1)$ on MEG topography and magnitude. This is in agreement with the results of (van den Broek et al., 1998) in a realistic FE head model and with the generally accepted idea that volume currents in the skull layer provide negligible contributions to the magnetic field (Hämäläinen and Sarvas, 1987). The effect of white matter anisotropy was, by contrast, negligible with an RDM of only about $5 \%$ and a MAG close to 1.0 for a realistic anisotropy ratio of 1:10, observations which agree well with those of Haueisen et al.(Haueisen et al., 2002). Note here, that only $15 \%$ of the finite elements 
in the vicinity of the somatosensory source were labeled as white matter and, following the results of (Haueisen et al., 2000; Gencer and Acar, 2004), we would expect a much larger influence for sources (even for eccentric ones) which are closer to or which are even embedded in an anisotropic medium.

For a superficial and radially oriented source, the EEG results for skull anisotropy agree well with the observations of others (Marin et al., 1998; van den Broek et al., 1998). With an RDM of about $11 \%$ and a MAG of about 0.5 , the influence on the potential topography was similar to that for the tangential dipole. The influence of skull anisotropy on the MEG was again minimal, in agreement with the reports of other groups (Hämäläinen and Sarvas, 1987; van den Broek et al., 1998). In our study, realistic white matter anisotropy only had a weak effect on the topography of the EEG (RDM $<5 \%)$, most likely because only few finite elements in the neighborhood of the source were assigned to the white matter compartment (Haueisen et al., 2000; Gencer and Acar, 2004). For the MEG, when compared to the error for the tangentially oriented source, RDM and MAG errors were twice as large, a result which again agrees with other reports (Haueisen et al., 2002) (our MEG results have to be compared to the flux density component $B_{y}$ in Table 2 of (Haueisen et al., 2002)). The large MEG topography error can be explained by the fact that white matter anisotropy influences the secondary (return) currents. The ratio of the secondary to the primary magnetic flux increases with increasing ratio of the radial to the tangential dipole orientation components (Haueisen, 1996).

The last simulated source was a deeper and therefore mainly radially oriented source in the left thalamus. In contrast to the superficial sources, there was a strong remote tissue anisotropy in the region between the source and the measurement sensors. From the line integral convolution visualization of the return currents, we found multiple areas where the main fiber direction and the return current vector in the model with anisotropic white matter compartment are highly parallel with highest degrees of parallelity within the bigger white matter fiber bundles, e.g., the left and right pyramidal tracts. In the isotropic case, the return currents are smoothly dipolar in shape, but in the anisotropic case the fiber geometry influences the flow to be largely parallel to the white matter fiber tracts. Thus for deeper sources, the leading cause for topography error was no longer the anisotropy of the skull but that of the white matter compartment. With an RDM of more than $15 \%$ for the MEG and more than $10 \%$ for the EEG and a MAG of about 0.7 , the effect of white matter anisotropy should not be neglected.

We have presented here the effect of remote anisotropy, i.e., in which the thalamus was modeled as an isotropic structure. Our reasoning was that the thalami are part of the human gray matter compartment (Shimony et al., 1999). Nevertheless, most histological methods identify 14 functionally specific anisotropic 
thalamic clusters referred to as nuclei (Buren and Borke, 1972). Recently it was shown that DT-MRI can noninvasively resolve the fiber orientation of those nuclei, using an automatic segmentation method (Wiegell et al., 2003). Therefore, in an even more realistic volume conductor model, the thalamus by itself would have to be considered as anisotropic gray matter tissue. Furthermore, the whole cortex is known to have an anisotropy ratio of about 1:2 (Nicholson and Freeman, 1975). If we then take into account that local conductivity changes in the vicinity of the sources have a large effect on EEG and MEG (Haueisen et al., 2000; Gencer and Acar, 2004), then the errors might be substantially larger than those presented in this study.

Our visualization results also showed the importance of the CSF compartment in determining bioelectric fields. Because of its high conductivity, the return current in this layer was much more distinct than in the rest of the head model so that it can be seen as a compartment with a strong "current distribution" effect. Because the conductivity of the human CSF is known quite accurately (Baumann et al., 1997), this result further underscores the importance of realistic high resolution finite element head modeling when compared to boundary or spherical head modeling.

We conclude that with the new visualization techniques for return current flow in high-resolution FE models, presented in our paper, insight is gained into the effect of tissue anisotropy, which is now more easily accessible. One implicit premise of our study was that if anisotropy affects the accuracy of the forward solution it will have at least as strong an influence over solutions to the associated inverse problem, which will be examined in a consequent paper (Anwander et al., 2002, 2005). We summarize that the modeling of skull anisotropy is important for EEG and can be neglected for MEG studies. Our results suggest, that the exact representation of the CSF compartment and the modeling of gray and white matter anisotropy is important for both EEG and MEG based reconstruction of the neural sources. Concerning white matter anisotropy, this is especially true with regard to the reconstruction of the orientation and strength components of the sources in the associated EEG and MEG inverse problem. The more the source is surrounded by anisotropy, the larger the influence. Recent developments for the finite element method in EEG/MEG source reconstruction (Weinstein et al., 2000; Wolters et al., 2002, 2004b; Gencer and Acar, 2004) dramatically reduce the complexity of the computations, so that the main disadvantage of FE modeling no longer exists and such modeling even with very high resolutions is now practical. 


\section{Acknowledgments}

The authors would like to thank A.Friederici from the MPI for Human Cognitive and Brain Sciences Leipzig, E.Zeidler and W.Hackbusch from the MPI for Mathematics in the Sciences and Seok Lew and Chris Johnson from the SCI Institute in Salt Lake City for their support and the many important and helpful comments. This work was supported by the MPI for Mathematics in the Sciences, Leipzig, by the IST-program of the European Community, project SIMBIO (http: //www. simbio.de) and the NIH NCRR center for Bioelectric Field Modeling, Simulation and Visualization (http://www.sci.utah.edu/ncrr).

\section{References}

Akhtari, M., Bryant, H., Marmelak, A., Flynn, E., Heller, L., Shih, J., Mandelkern, M., Matlachov, A., Ranken, D., Best, E., DiMauro, M., Lee, R., Sutherling, W., 2002. Conductivities of three-layer live human skull. Brain Top. 14 (3), 151-167.

Anwander, A., Wolters, C., Dümpelmann, M., Knösche, T., 2002. Influence of realistic skull and white matter anisotropy on the inverse problem in EEG/MEG-source localization. In: H. Nowak, J. Haueisen, F. Giessler and R. Huonker, BIOMAG2002, Proc. of the 13th Int. Conf. on Biomagnetism, VDE Verlag GmbH, Berlin, Offenbach, URL http://biomag2002. uni-jena.de, 2002, pp. 679-681.

Anwander, A., Wolters, C., Dümpelmann, M., Knösche, T., 2005. Influence of realistic skull and white matter anisotropy on the inverse problem in EEG/MEG-source localization, in preparation.

Basser, P., Bihan, D. L., Mattiello, J., 1994a. Estimation of the effective selfdiffusion-tensor from the NMR spin echo. J. Magn. Res. B 103, 247-254.

Basser, P., Mattiello, J., LeBihan, D., 1994b. MR diffusion tensor spectroscopy and imaging. Biophys. J. 66, 259-267.

Basser, P., Pierpaoli, C., 1996. Microstructural and physiological features of tissues elucidated by quantitive-diffusion-tensor MRI. J. Magn. Reson. B 111, 209-219.

Baumann, S., Wozny, D., Kelly, S., Meno, F., 1997. The electrical conductivity of human cerebrospinal fluid at body temperature. IEEE Trans Biomed. Eng. 44 (3), 220-223.

BioPSE, 2002. Problem Solving Environment for modeling, simulation, and visualization of bioelectric fields. Scientific Computing and Imaging Institute (SCI), URL http://software.sci.utah.edu/biopse.html.

Bruno, P., Vatta, F., Minimel, S., Inchingolo, P., 2004. Referenced EEG and head volume conductor model: Geometry and parametrical setting. In: Proc. of the 26th Annual Int. Conf. IEEE Engineering in Medicine and Biology 
Society. San Francisco, USA, Sep. 1-5, URL http://www.ucsfresno.edu/ embs 2004.

Buchner, H., Knoll, G., Fuchs, M., Rienäcker, A., Beckmann, R., Wagner, M., Silny, J., Pesch, J., 1997. Inverse localization of electric dipole current sources in finite element models of the human head. Electroenc. Clin. Neurophysiol. 102, 267-278.

Buren, J. V., Borke, R., 1972. Variations and Connections of the Human Thalamus. 2.Springer-Verlag New York.

Cabral, B., Leedom, L. C., 1993. Imaging vector fields using line integral convolution. In: Proceeding of SIGGRAPH 1993 Conference. pp. 263-272.

Cuffin, B., 1996. EEG localization accuracy improvements using realistically shaped head models. IEEE Trans Biomed. Eng. 43, 299-303.

CURRY, 2000. CURrent Reconstruction and Imaging, URL http://www. neuro.com.

de Munck, J., 1988. The potential distribution in a layered anisotropic spheroidal volume conductor. J. Appl. Phys. 64, 465-469.

Garth, C., Tricoche, X., Salzbrunn, T., Bobach, T., Scheuermann, G., May 2004. Surface techniques for vortex visualization. In: Proceedings of Joint Eurographics - IEEE TCVG Symposium on Visualization. p. (to appear). URL http://www.sci.utah.edu/publications/garth04/vissym04.pdf

Geddes, L., Baker, L., 1967. The specific resistance of biological material. A compendium of data for the biomedical engineer and physiologist. Med.Biol.Eng. 5, 271-293.

Gencer, N., Acar, C., 2004. Sensitivity of EEG and MEG measurements to tissue conductivity. Phys.Med.Biol. 49, 701-717.

Haase, A., Frahm, J., Matthaei, D., Hänicke, W., Merboldt, K.-D., 1986. FLASH imag.: rapid NMR imag. using low flip-angle pulses. J.Magn.Reson. 67, 258-266.

Hämäläinen, M., Sarvas, J., 1987. Feasibility of the homogeneous head model in the interpretation of neuromagnetic fields. Phys.Med.Biol. 32, 91-97.

Haueisen, J., 1996. Methods of numerical field calculation for neuromagnetic source localization. Ph.D. thesis, Shaker-Verlag Aachen, ISBN 3-8265-16915.

Haueisen, J., Ramon, C., Brauer, H., Nowak, H., 2000. The influence of local conductivity changes on MEG and EEG. Biomedizinische Technik 45 (7-8), $211-214$.

Haueisen, J., Tuch, D., Ramon, C., Schimpf, P., Wedeen, V., George, J., Belliveau, J., 2002. The influence of brain tissue anisotropy on human EEG and MEG. NeuroImage 15, 159-166.

Huiskamp, G., Vroeijenstijn, M., van Dijk, R., Wieneke, G., van Huffelen, A., 1999. The need for correct realistic geometry in the inverse EEG problem. IEEE Trans Biomed. Eng. 46 (11), 1281-1287.

Koch, M., 2000. Measurement of the self-diffusion tensor of water in the human brain. Ph.D. thesis, Univ. of Leipzig, Germany.

Law, S., 1993. Thickness and resistivity variations over the upper surface of 
the human skull. Brain Top. 2, 99-109.

Lee, J., Garwood, M., Menon, R., Adriany, G., Anderson, P., Truwit, C., Ugurbil, K., 1995. High contrast and fast 3D magnetic resonance imaging at high fields. Magn.Reson.Med. 34, 308-312.

Marin, G., Guerin, C., Baillet, S., Garnero, L., G., M., 1998. Influence of skull anisotropy for the forward and inverse problem in EEG: simulation studies using the FEM on realistic head models. Human Brain Mapping 6, 250-269.

Meijs, J., Weier, O., Peters, M., van Oosterom, A., 1989. On the numerical accuracy of the boundary element method. IEEE Trans Biomed. Eng. 36, $1038-1049$.

Mohr, M., Vamrunste, B., 2003. Comparing iterative solvers for linear systems associated with the finite difference discretisation of the forward problem in electro-encephalographic source analysis. Med. Biol. Eng. Comp. 41, 75-84.

NeuroFEM, 2000-2005. A parallel MPI-based C++ class structured code for fast high-resolution EEG and MEG forward modeling. A. Anwander and C.H. Wolters SimBio (2000-2003).

Nicholson, C., Freeman, J., 1975. Experimental optimization of current sourcedensity technique for anuran cerebellum. J. Neurophysiol. 38 (2), 369-382.

Nicholson, P., 1965. Specific impedance of cerebral white matter. Exp.Neurol. 13, 386-401.

Norris, D., Börnert, P., 1993. Coherence and interference in ultra-fast RARE experiments. J. Magn. Reson. A 105, 123-127.

Ollikainen, J., Vauhkonen, M., Karjalainen, P., Kaipio, J., 1999. Effects of local skull inhomogeneities on EEG source estimation. Med.Eng.Phys. 21, $143-154$.

Pajevic, S., Pierpaoli, C., 1999. Color schemes to represent the orientation of anisotropic tissues from diffusion tensor data: application to white matter fiber tract mapping in the human brain. Magn.Reson.Med. 42, 526-540.

Peters, M., de Munck, J., 1991. The influence of model parameters on the inverse solution based on MEGs and EEGs. Acta Otolaryngol [Suppl] (Stockh) 491, 61-69.

Pham, D., Prince, J., 1999. An adaptive fuzzy C-means algorithm for image segmentation in the presence of intensity inhomogeneities. Pat. Rec. Let. 20, 57-68.

Pohlmeier, R., Buchner, H., Knoll, G., Rienäcker, A., Beckmann, R., Pesch, J., 1997. The influence of skull-conductivity misspecification on inverse source localization in realistically shaped finite element head models. Brain Top. 9 (3), 157-162.

Ramon, C., Schimpf, P., Haueisen, J., Holmes, M., Ishimaru, A., 2004. Role of soft bone, CSF and gray matter in EEG simulations. Brain Topography 16 (4), 245-248.

Roth, B., Balish, M., Gorbach, A., Sato, S., 1993. How well does a three-sphere model predict positions of dipoles in a realistically shaped head. Electroenc. Clin. Neurophysiol. 87, 175-184.

Rush, S., Driscoll, D., 1968. Current distribution in the brain from surface 
electrodes. Anesthesia and analgetica 47 (6), 717-723.

Sarvas, J., 1987. Basic mathematical and electromagnetic concepts of the biomagnetic inverse problem. Phys.Med.Biol. 32 (1), 11-22.

Schimpf, P., Ramon, C., Haueisen, J., 2002. Dipole models for the EEG and MEG. IEEE Trans Biomed. Eng. 49 (5), 409-418.

Shimony, J. S., McKinstry, R., E.Akbudak, J.A.Aronovitz, A.Z.Snyder, N.F.Lori, T.S.Cull, T.E.Conturo, 1999. Quantitative diffusion-tensor anisotropy brain MR imaging: Normative human data and anatomic analysis. Radiology 212, 770-784.

SimBio, 2000-2003. SimBio: A Generic Environment for Bio-Numerical Simulation, IST-Program of the European Commission, Project No.10378, URL http://www.simbio.de.

Tuch, D., Wedeen, V., Dale, A., George, J., Belliveau, J., 2001. Conductivity tensor mapping of the human brain using diffusion tensor MRI. Proc.Natl.Acad.Sci.USA 98 (20), 11697-11701.

van den Broek, S., Reinders, F., Donderwinkel, M., Peters, M., 1998. Volume conduction effects in EEG and MEG. Electroenc. Clin. Neurophysiol. 106, $522-534$.

Wagner, M., 1998. Rekonstruktion neuronaler Ströme aus bioelektrischen und biomagnetischen Messungen auf der aus MR-Bildern segmentierten Hirnrinde. Ph.D. thesis, Shaker-Verlag Aachen, ISBN 3-8265-4293-2.

Wang, Y., Haynor, D., Kim, Y., 2001. An investigation of the importance of myocardial anisotropy in finite-element modeling of the heart: Methodology and application to the estimation of defibrillation efficacy. IEEE Trans Biomed. Eng. 48 (12).

Weinstein, D., Zhukov, L., Johnson, C., 2000. Lead-field bases for electroencephalography source imaging. Annals of Biomed.Eng. 28 (9), 1059-1066.

Wiegell, M., Tuch, D., Larsson, H., Wedeen, V., 2003. Automatic segmentation of thalamic nuclei from diffusion tensor magnetic resonance imaging. NeuroImage 19, 391-401.

Wolters, C., 2003. Influence of Tissue Conductivity Inhomogeneity and Anisotropy on EEG/MEG based Source Localization in the Human Brain. No. 39 in MPI Series in Cognitive Neuroscience. MPI of Cognitive Neuroscience Leipzig, iSBN 3-936816-11-5 (also: Leipzig, Univ., Diss., URL http://dol . uni-leipzig.de/pub/2003-33).

Wolters, C., Kuhn, M., Anwander, A., Reitzinger, S., 2002. A parallel algebraic multigrid solver for finite element method based source localization in the human brain. Comp.Vis.Sci. 5 (3), 165-177.

Wolters, C., Reitzinger, S., Basermann, A., Burkhardt, S., Hartmann, U., Kruggel, F., Anwander, A., 2000. Improved tissue modeling and fast solver methods for high resolution FE-modeling in EEG/MEG-source localization. In: J. Nenonen, R.J. Ilmoniemi and T. Katila,BIOMAG2000, Proc. of the 12th Int. Conf. on Biomagnetism, Helsinki Univ. of Tech., Finland, Aug.1317, URL http://biomag2000.hut.fi, 2000, pp. 655-658.

Wolters, C. H., Anwander, A., Reitzinger, S., Haase, G., 2004a. Algebraic 
multigrid with multiple right-hand-side treatment for an efficient computation of EEG and MEG lead field bases. In: E. Halgren, S. Ahlfors, M. Hämäläinen and D. Cohen, BIOMAG2004, Proc. of the 14th Int. Conf. on Biomagnetism", Boston, USA, Aug.8-12, URL http://www. biomag2004. org, 2004, pp. 465-466.

Wolters, C. H., Grasedyck, L., Hackbusch, W., 2004b. Efficient computation of lead field bases and influence matrix for the FEM-based EEG and MEG inverse problem. Inverse Problems 20 (4), 1099-1116. 
List of Captions for the Tables

Table 1: Simulated values for the skull conductivity tensor eigenvalues: The ratio was given and the eigenvalues were computed under the respective constraint. 


\section{List of Captions for the Figures}

Figure 1: Five tissue head model: The result of the segmentation in axial (left), coronal (middle) and sagittal (right) view. The color labels correspond to: yellow - white matter, dark blue - gray matter, light blue - CSF, green - skull, brown - skin.

Figure 2: Visualization of the Fractional Anisotropy (FA) of the DT-MRI measurements in the white matter compartment. The first row shows the FA values in red-yellow-white color scale overlaid on the T1-MRI. The second row shows the orientation of the principal tensor eigenvector in color-coding according to the red-green-blue sphere (shown in the left figure) with red indicating mediolateral, green anteroposterior and blue superoinferior direction. The brightness of the color is scaled to the FA (max. 0.75). The white matter fiber orientation map is overlaid on the T1-MRI.

Figure 3: Sagittal cut through the five tissue tetrahedra model (color labeling like in Figure 1). For visualization, the software tool SimBio (2000-2003)-VM (VM: Visualization Module) was used.

Figure 4: Visualization of the computed surface for the determination of radial skull anisotropy directions onto the underlying T1-MRI.

Figure 5: Conductivity tensor ellipsoids in the barycenters of the tetrahedra elements: Normalized and colored (by trace) for 1:2 (vol.const.) skull and white matter anisotropy. The highest trace values can be found in the CSF compartment (red) and the lowest in the skull compartment (dark blue). Note the mainly top-bottom fiber directions of the pyramidal tracts and the mainly left-right orientation over the corpus callosum. Tensor validation and visualization was carried out using the software BioPSE (2002).

Figure 6: Tangentially (top row) and radially (middle row) oriented somatosensory source and deep thalamic source (bottom row): EEG and MEG topography error (left) and magnitude error (right) for different anisotropy ratios: For the EEG, errors due to anisotropy effects of skull, white matter and both 
skull and white matter are presented for the tensor volume retaining (Vol) and Wang's constraint (Wang). For MEG, only white matter anisotropy effects for both constraints are presented, because skull anisotropy was found to have no influence.

Figure 7: Linearly interpolated EEG isopotential lines (in a blue-white-red scale) and MEG isofield lines (in a rainbow scale) (top row) and isopotential surfaces for $-5 \mu V$ (blue), $0 \mu V$ (white) and $5 \mu V$ (red) (bottom row) for a mainly tangentially oriented source in somatosensory cortex: Isotropic model (Left), 1:10 anisotropic skull using the volume constraint (middle) and 1:10 anisotropic white matter using the volume constraint (right).

Figure 8: Visualization of return current surfaces for the mainly tangentially oriented source in somatosensory cortex for the isotropic model (left) and the model with 1:10 anisotropic skull compartment (right): In order to define a starting line for the flow integration, we divided the interval from highest to lowest surface potential for both isotropic and anisotropic model into 19 intervals (18 isopotential lines). The flow computation then started at the maximal and minimal isopotential lines for both models and integrated along the return current flow into the volume until close to the singularity of the primary current. We used the color of the surface isopotential value for the color coding of the corresponding flow surface.

Figure 9: Linearly interpolated EEG isopotential lines (in a blue-white-red scale) and MEG isofield lines (in a rainbow scale) (top row) and isopotential surfaces for $-1 \mu V$ and $7 \mu V$ (bottom row) for a mainly radially oriented source in somatosensory cortex: Isotropic model (Left), 1:10 anisotropic skull using the volume constraint (middle) and 1:10 anisotropic white matter using the volume constraint (right).

Figure 10: Linearly interpolated EEG isopotential lines (in a blue-white-red scale) and MEG isofield lines (in a rainbow scale) (top row) and isopotential surfaces for $-3 \mu V, 0 \mu V$ and $3 \mu V$ (bottom row) for a deep thalamic source: Isotropic model (Left), 1:10 anisotropic skull using the volume constraint (middle) and 1:10 anisotropic white matter using the volume constraint (right). 
Figure 11: Surface return current for the left thalamic source in the isotropic model and in the model with 1:10 anisotropic white matter compartment (volume constraint) visualized with the LIC technique. The magnitude of the return current is color coded. The direction is indicated by the texture.

Figure 12: Visualization of the return currents (thalamic source) within the white matter mask on a coronal slice passing through the thalamus overlaid on the T1-MRI for the isotropic model and the corresponding model with anisotropic white matter compartment (volume constraint): The projections of the current directions on the image plane are shown as black lines and the magnitude is color coded (two linear scales, one from 0.3 to $0.003 \mathrm{~A} / \mathrm{m}^{2}$ in the neighborhood of the source and the second from 0.003 to $0 \mathrm{~A} / \mathrm{m}^{2}$ for remote locations.

Figure 13: As a measure of the parallelity/similarity, the cosine of the angle between the main eigenvector of the conductivity tensor in anisotropic white matter (its projection onto the coronal plane is shown in black) and the return current vector (not shown here) is color coded within the white matter mask and overlaid on the T1-MRI for the isotropic model (top) and the corresponding model with anisotropic white matter compartment (volume constraint, bottom row).

Figure 14: Return currents for the left thalamic source on a coronal cut through the isotropic model (top row) and the model with 1:10 anisotropic white matter compartment (volume constraint, bottom row): The return current directions are indicated by the texture and the magnitude is color coded (the upper scale was limited to $0.02 \mathrm{~A} / \mathrm{m}^{2}$, see Figure 12 for the correct magnitude in the source area). 


\section{Tables}

\begin{tabular}{|r|rr|rr|rr|rr|}
\hline ratio & \multicolumn{3}{|c|}{ Skull tensor eigenvalues } & \multicolumn{3}{c|}{ White matter tensor eigenvalues } \\
\hline & \multicolumn{2}{|c|}{ Volume constraint } & \multicolumn{2}{|c|}{ Wang's constraint } & Volume constraint & Wang's constraint \\
\hline & $\sigma^{\text {rad }}$ & $\sigma^{\text {tang }}$ & $\sigma^{\text {rad }}$ & $\sigma^{\text {tang }}$ & $\sigma^{\text {trans }}$ & $\sigma^{\text {long }}$ & $\sigma^{\text {trans }}$ & $\sigma^{\text {long }}$ \\
\hline $1: 1$ (iso) & 0.0042 & 0.0042 & 0.0042 & 0.0042 & 0.14 & 0.14 & 0.14 & 0.14 \\
$1: 2$ & 0.0026 & 0.0053 & 0.003 & 0.0058 & 0.111 & 0.222 & 0.099 & 0.19798 \\
$1: 5$ & 0.00143 & 0.0072 & 0.00188 & 0.00938 & 0.0818 & 0.41 & 0.0626 & 0.31309 \\
$1: 10$ & 0.000905 & 0.00905 & 0.00133 & 0.01326 & 0.065 & 0.65 & 0.04427 & 0.4427 \\
$1: 100$ & 0.000195 & 0.0195 & 0.00042 & 0.042 & 0.03016 & 3.016 & 0.014 & 1.4 \\
\hline
\end{tabular}

Table 1 
Figures

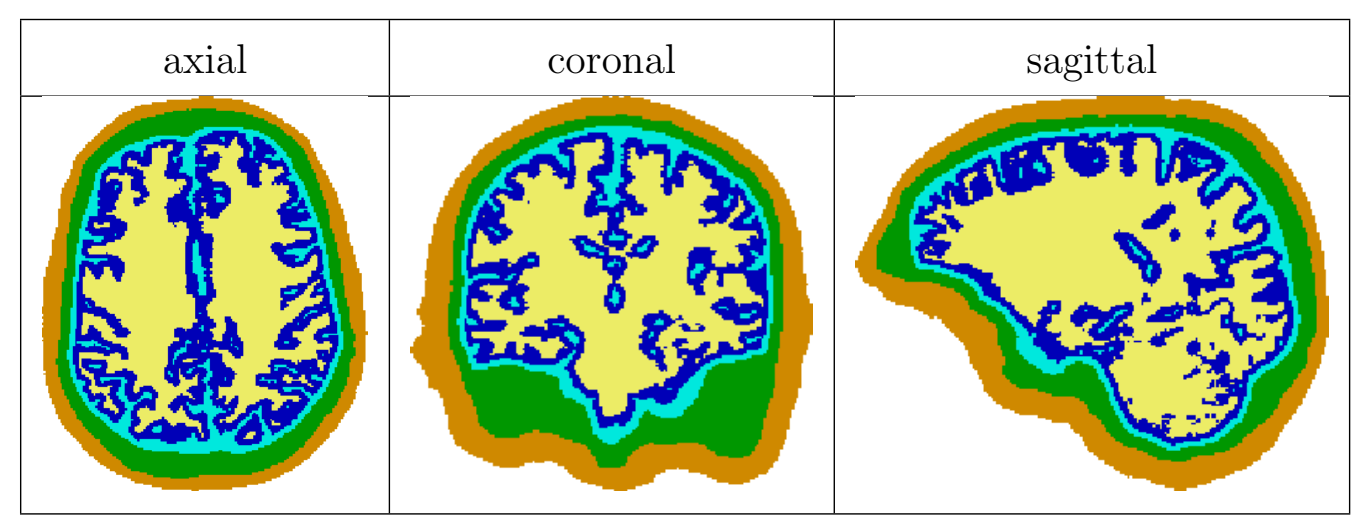

Figure 1.

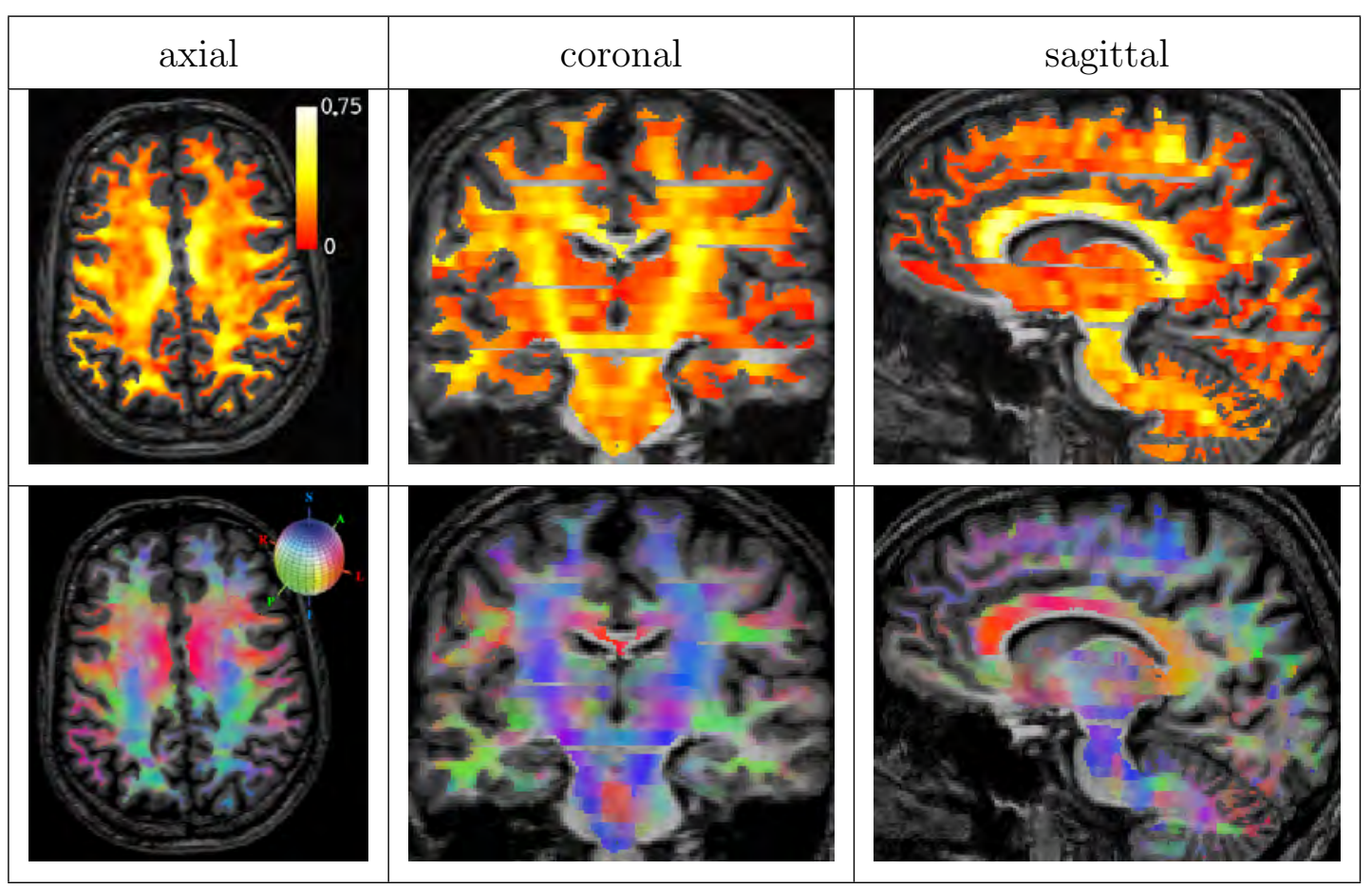

Figure 2. 


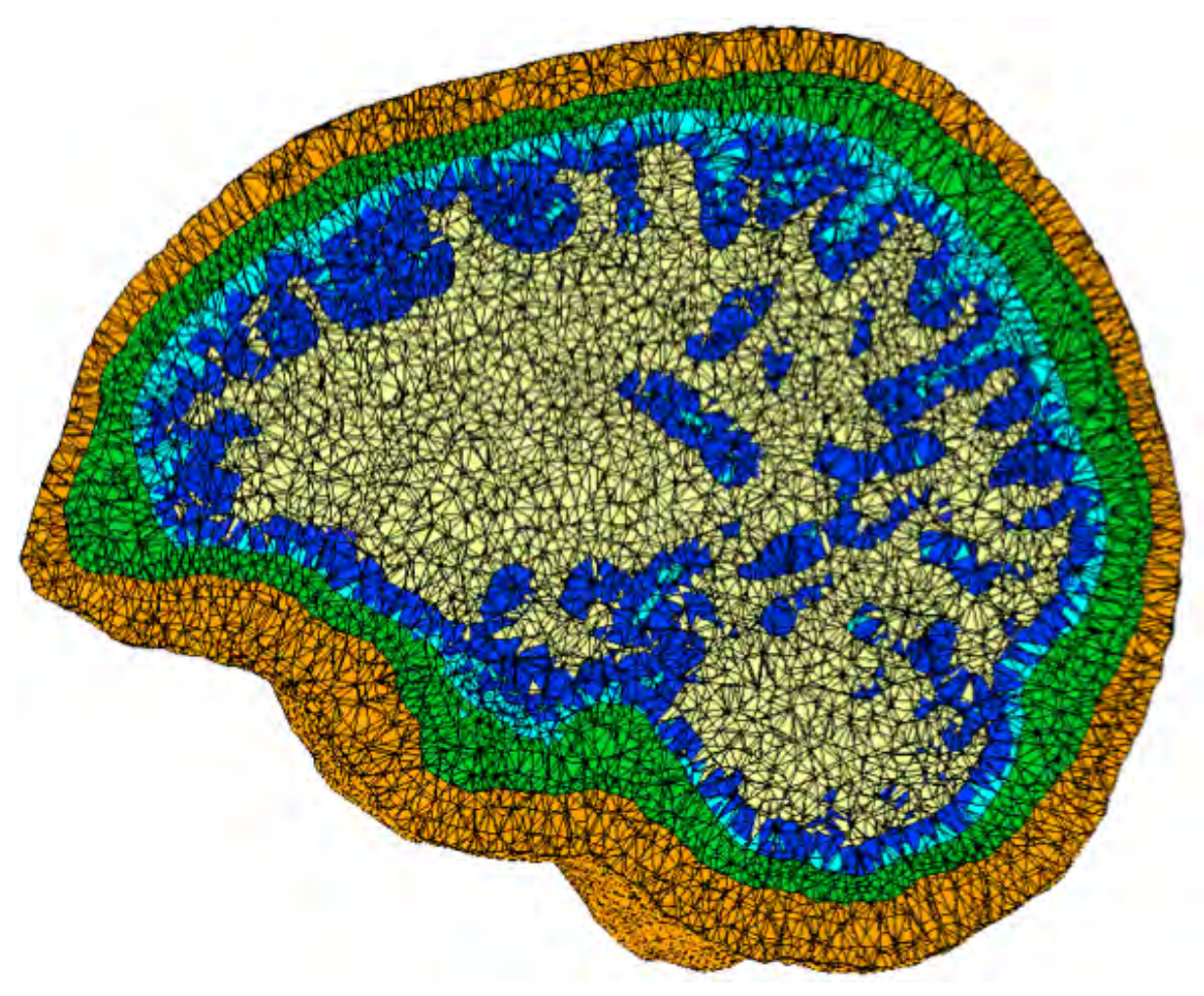

Figure 3.
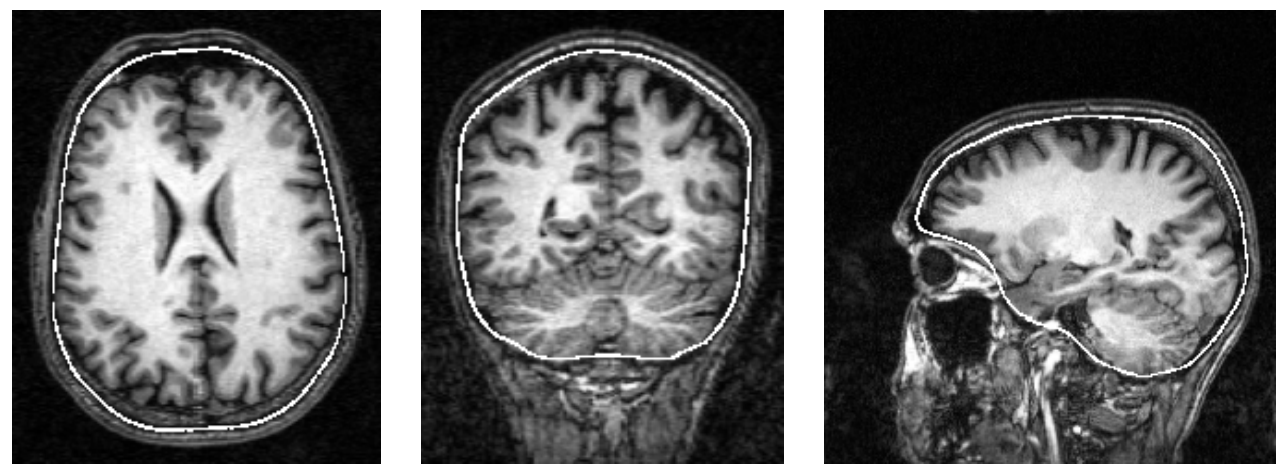

Figure 4. 


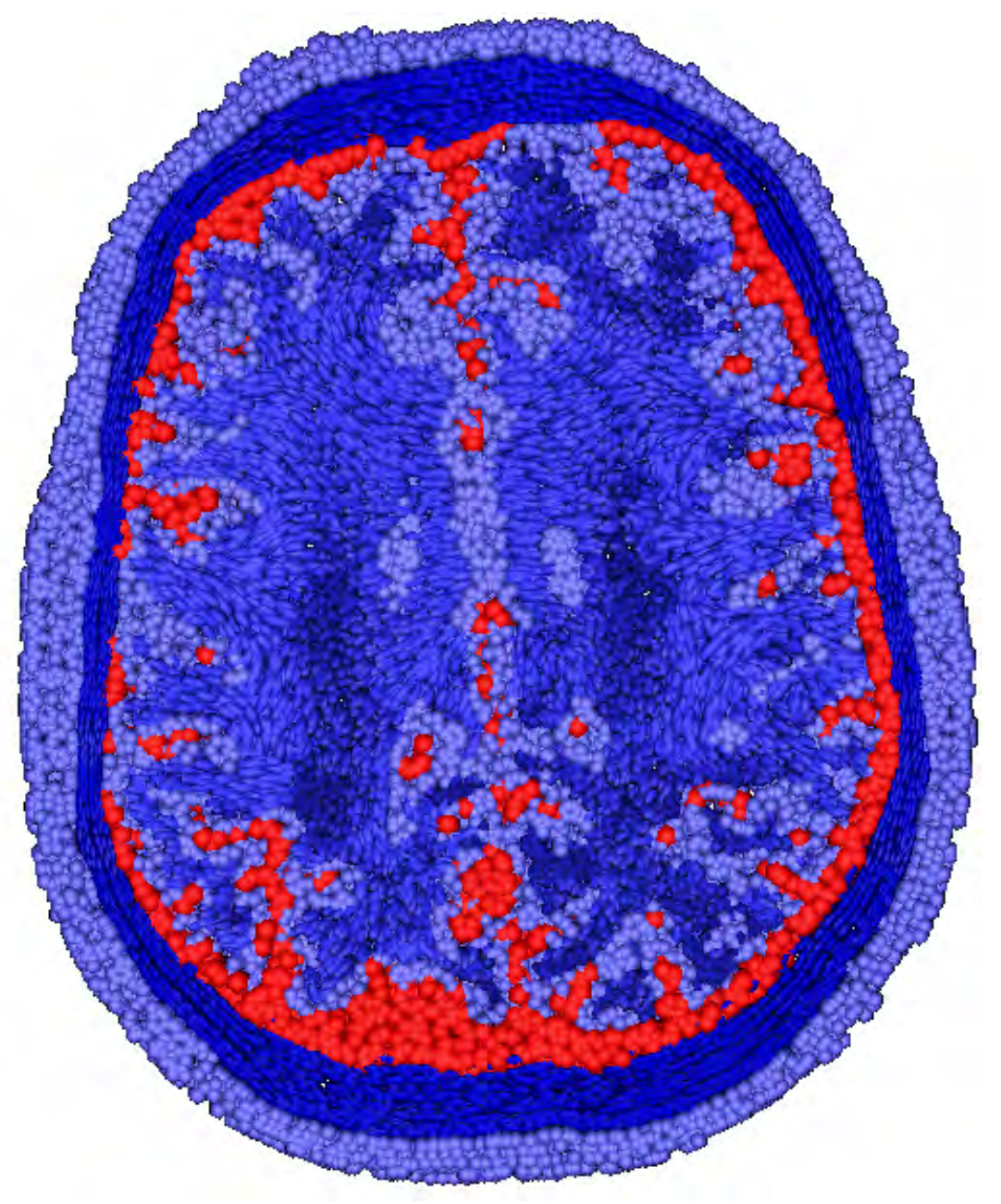

Figure 5. 
Tangentially oriented somatosensory source
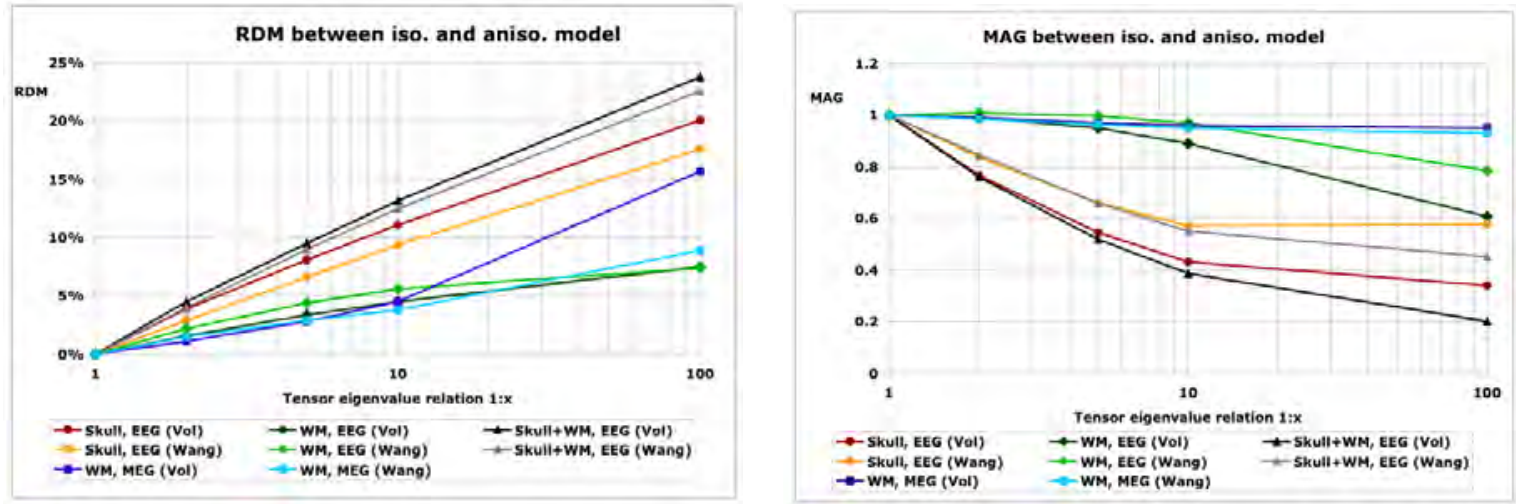

Radially oriented somatosensory source
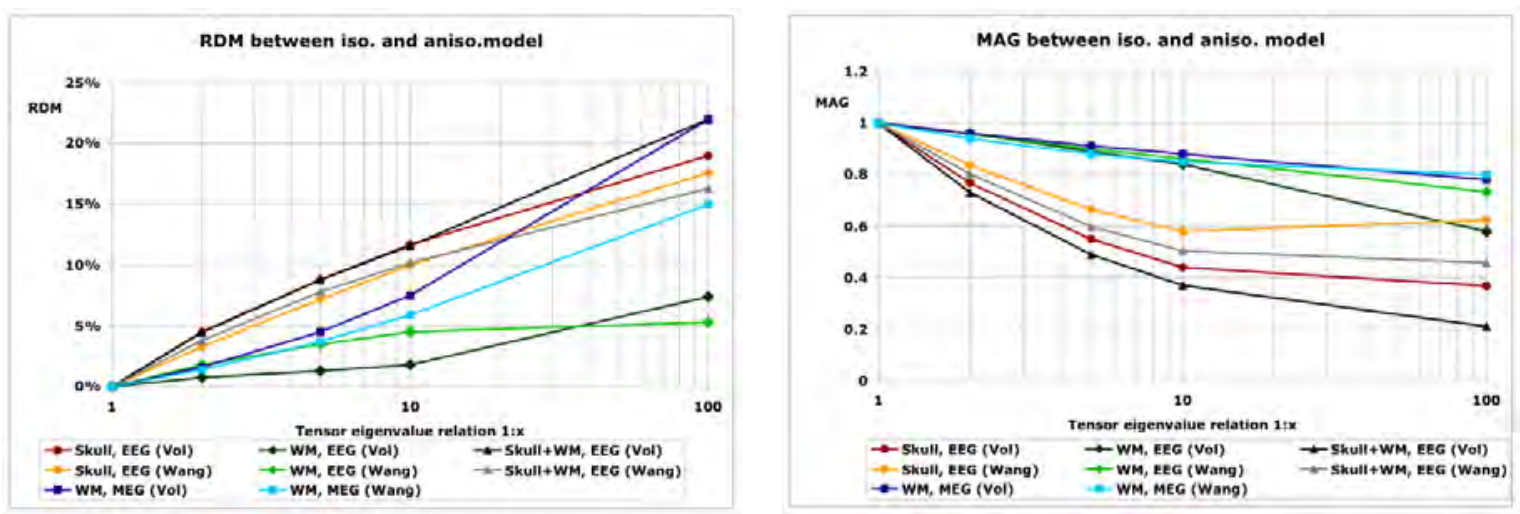

Deep thalamic source
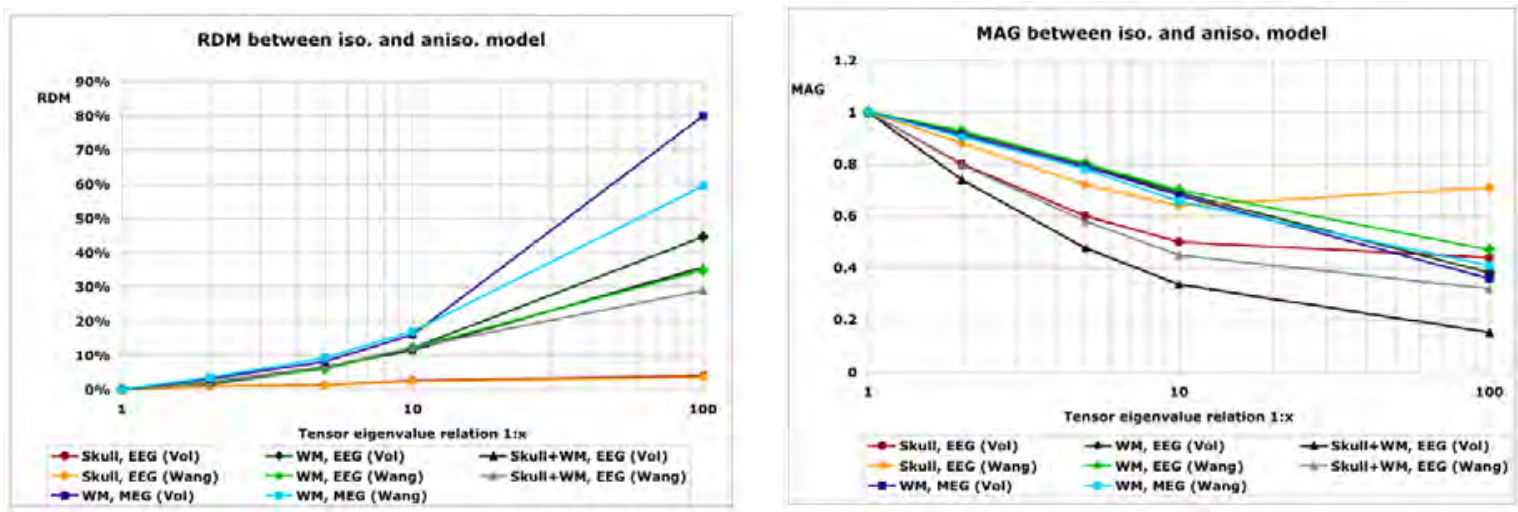

Figure 6 . 


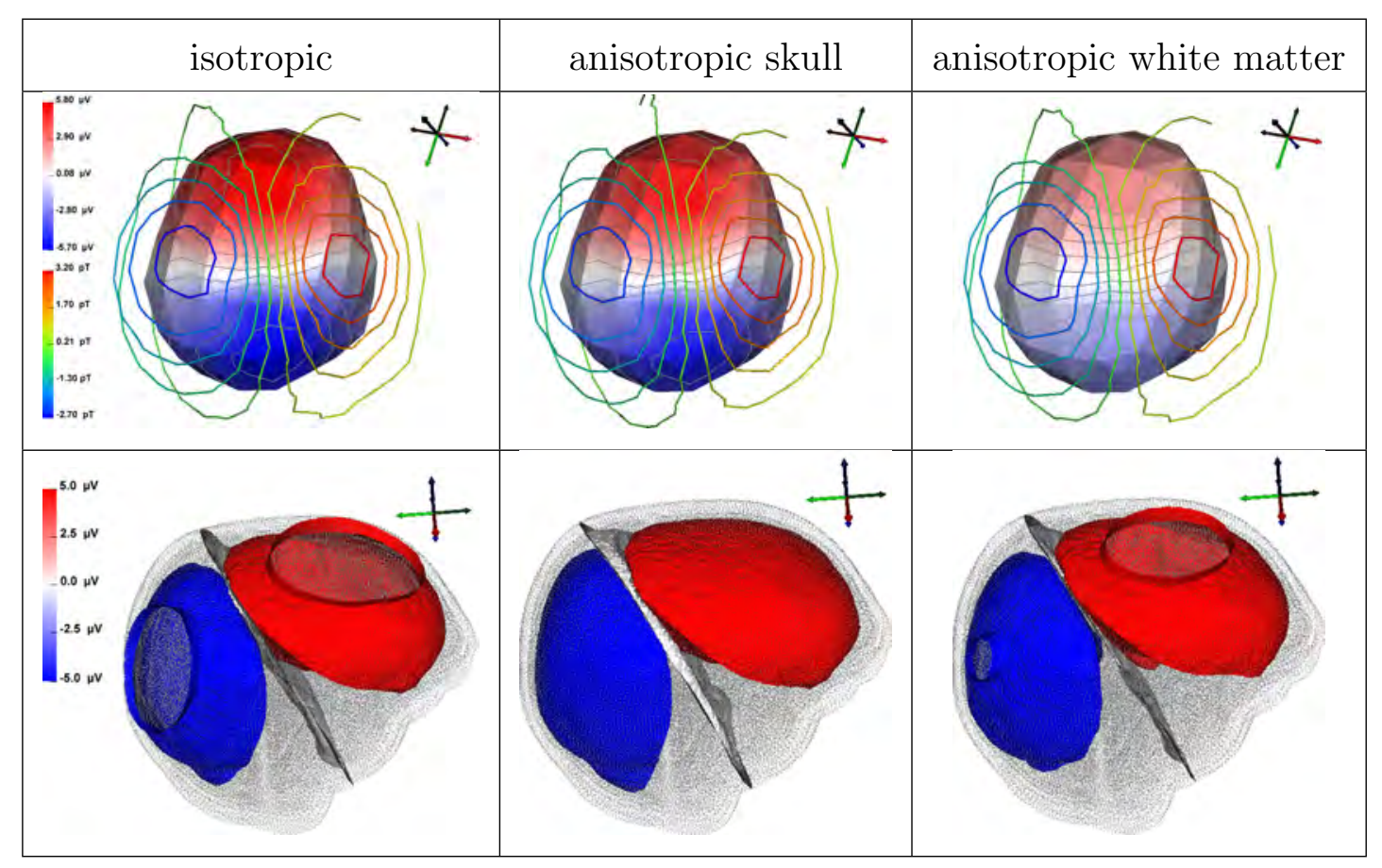

Figure 7.

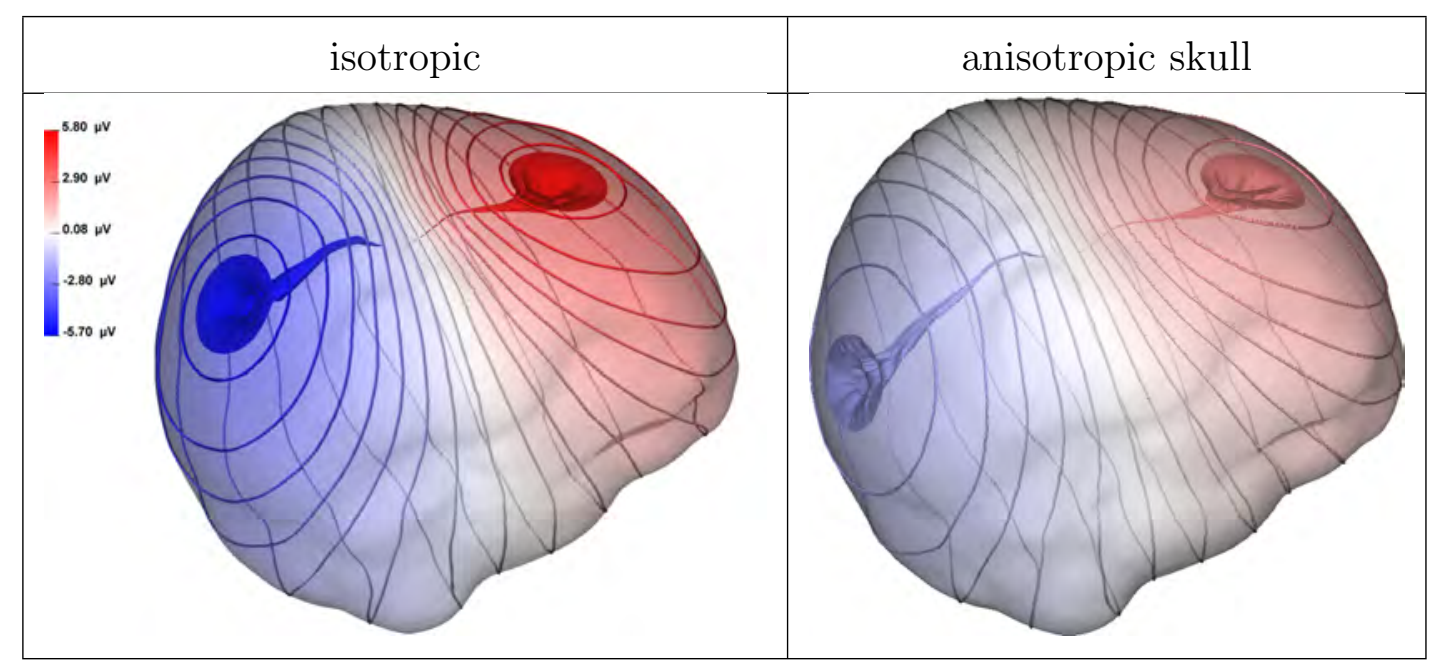

Figure 8. 


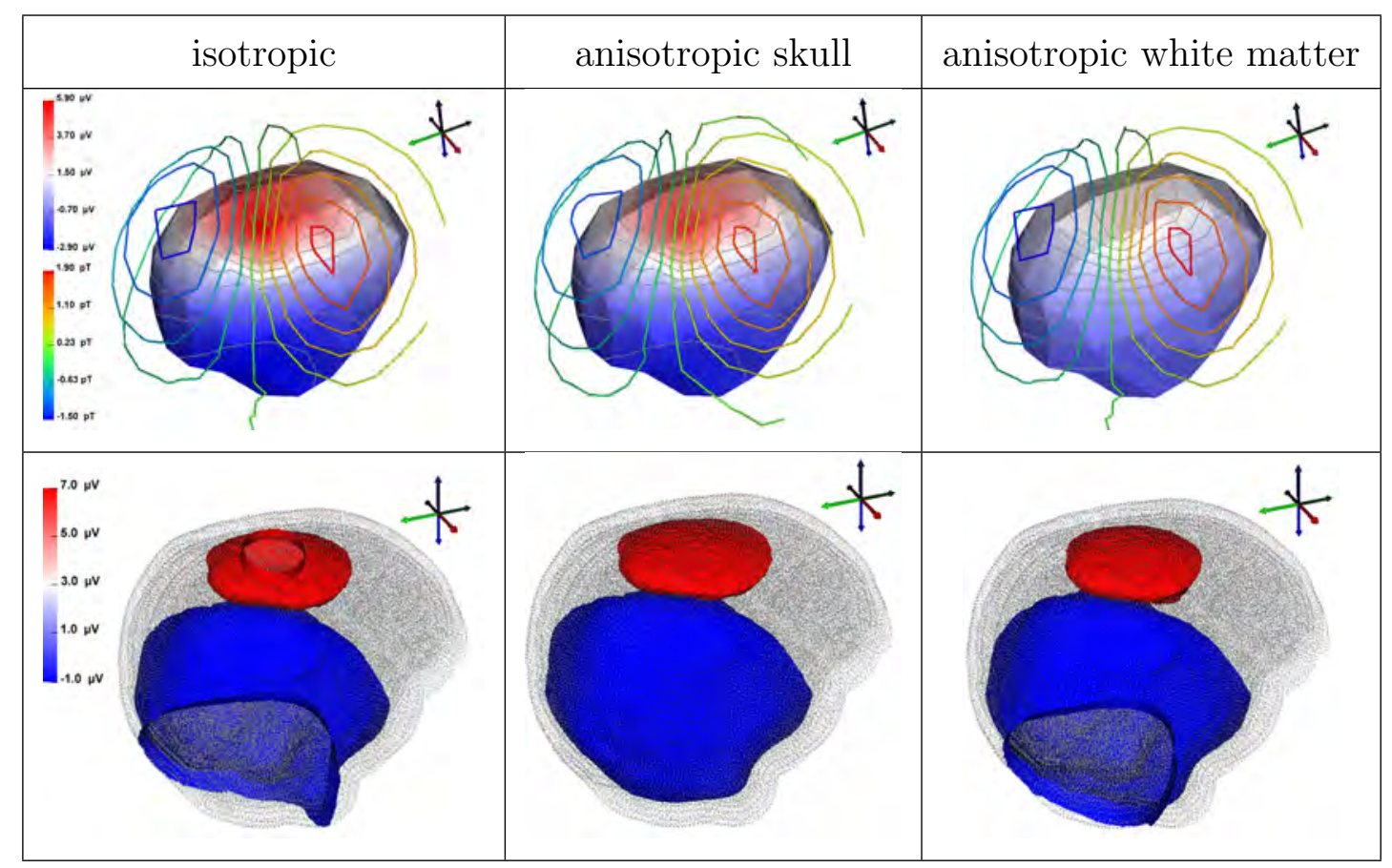

Figure 9.

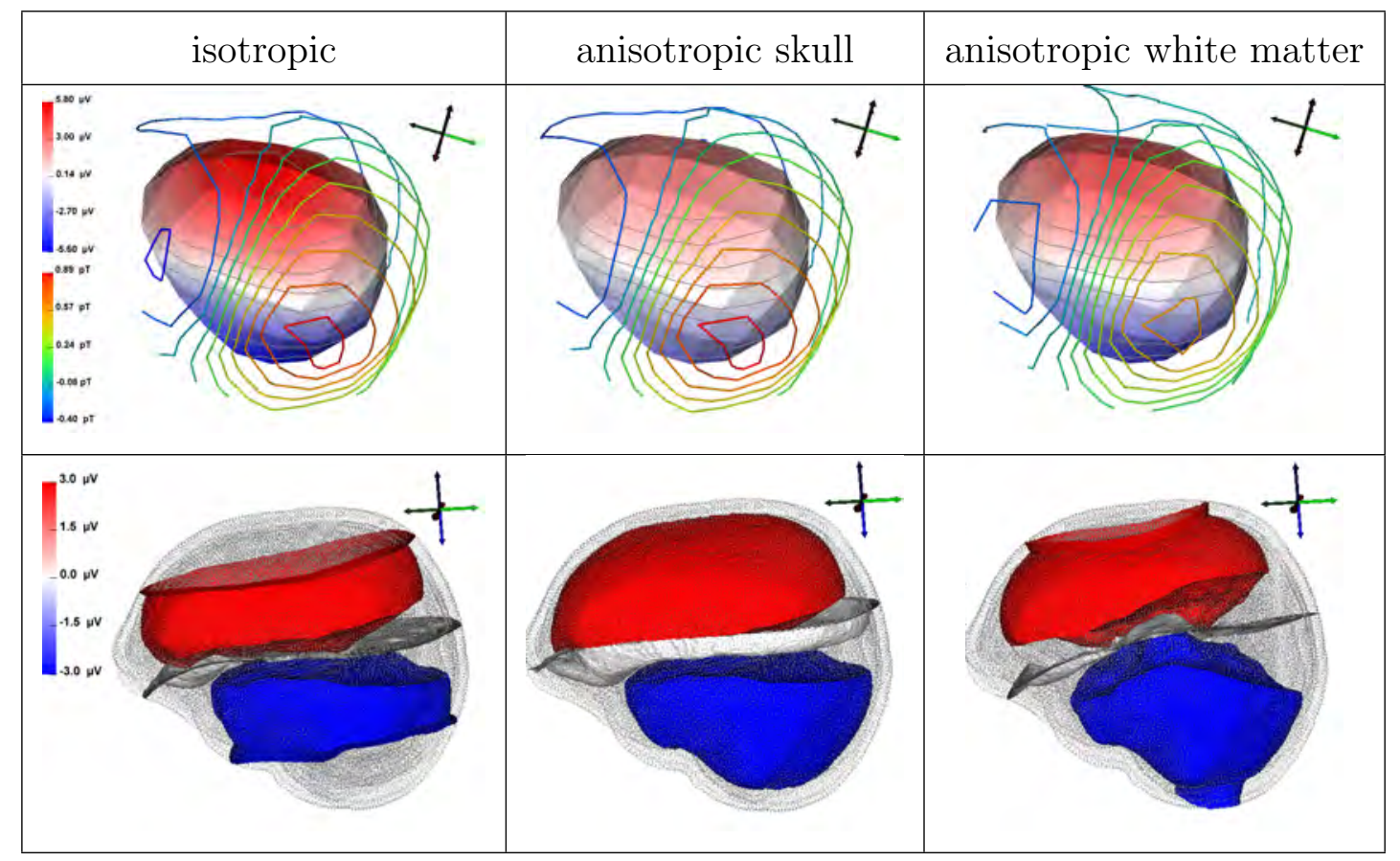

Figure 10. 


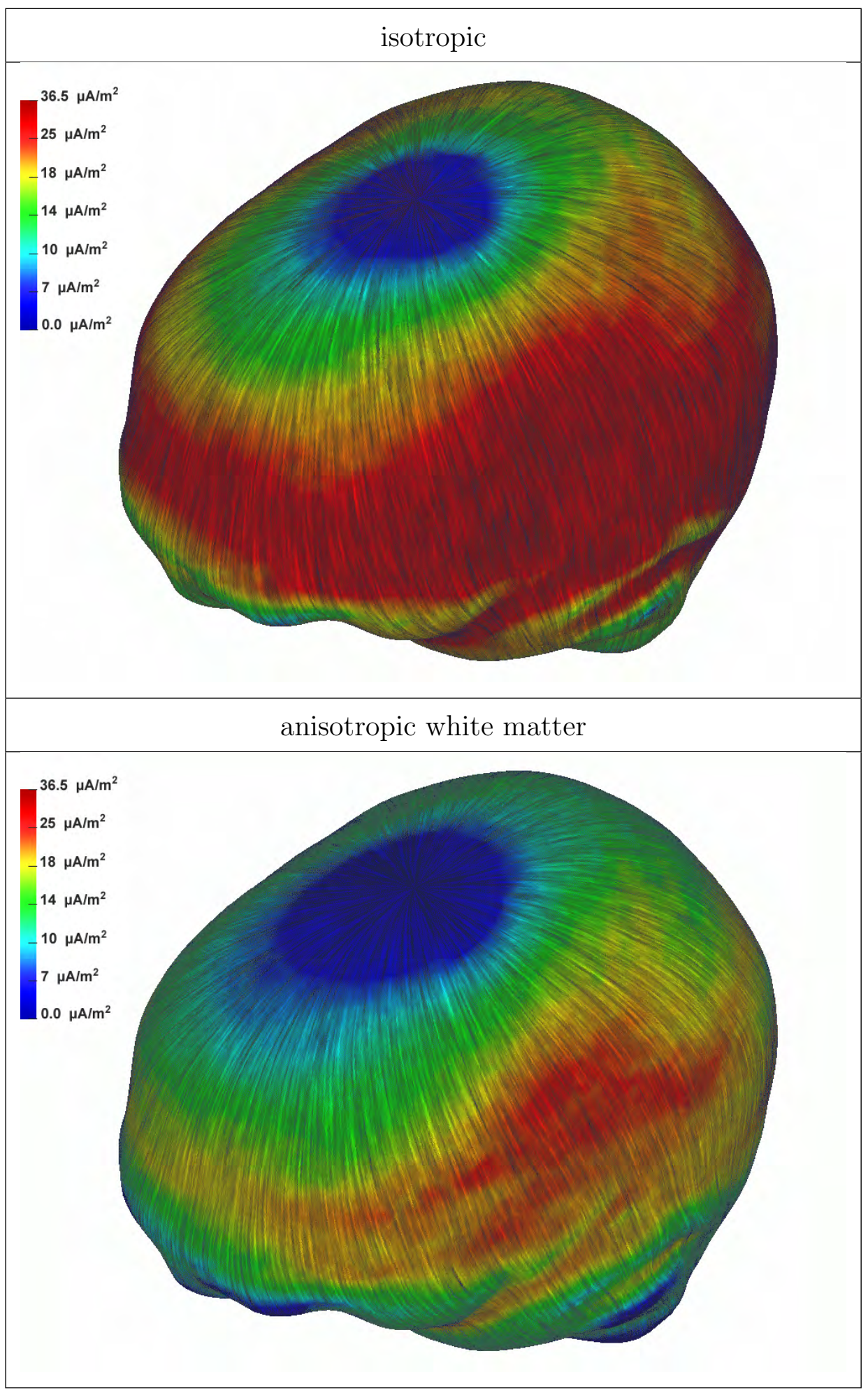

Figure 11. 


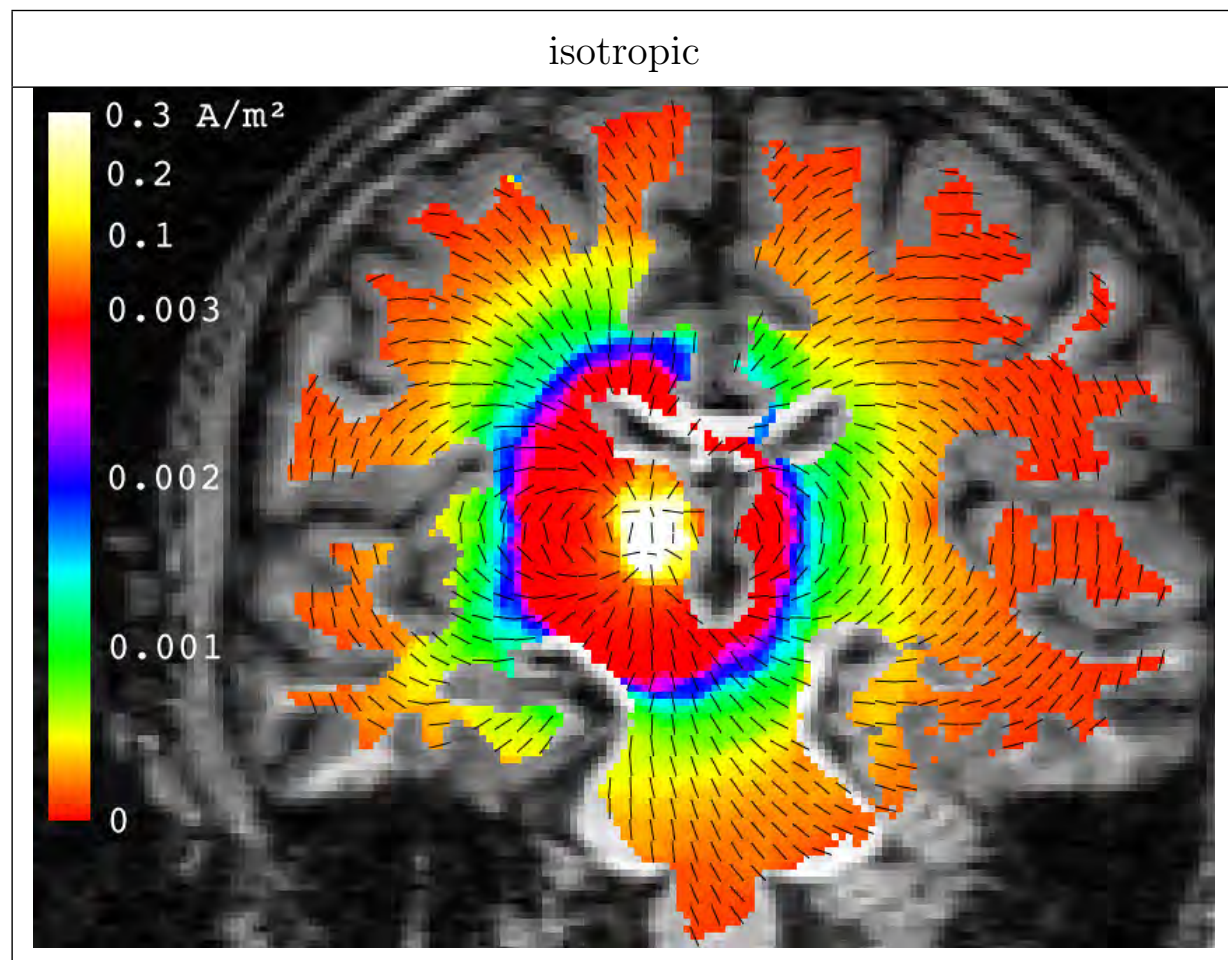

anisotropic white matter

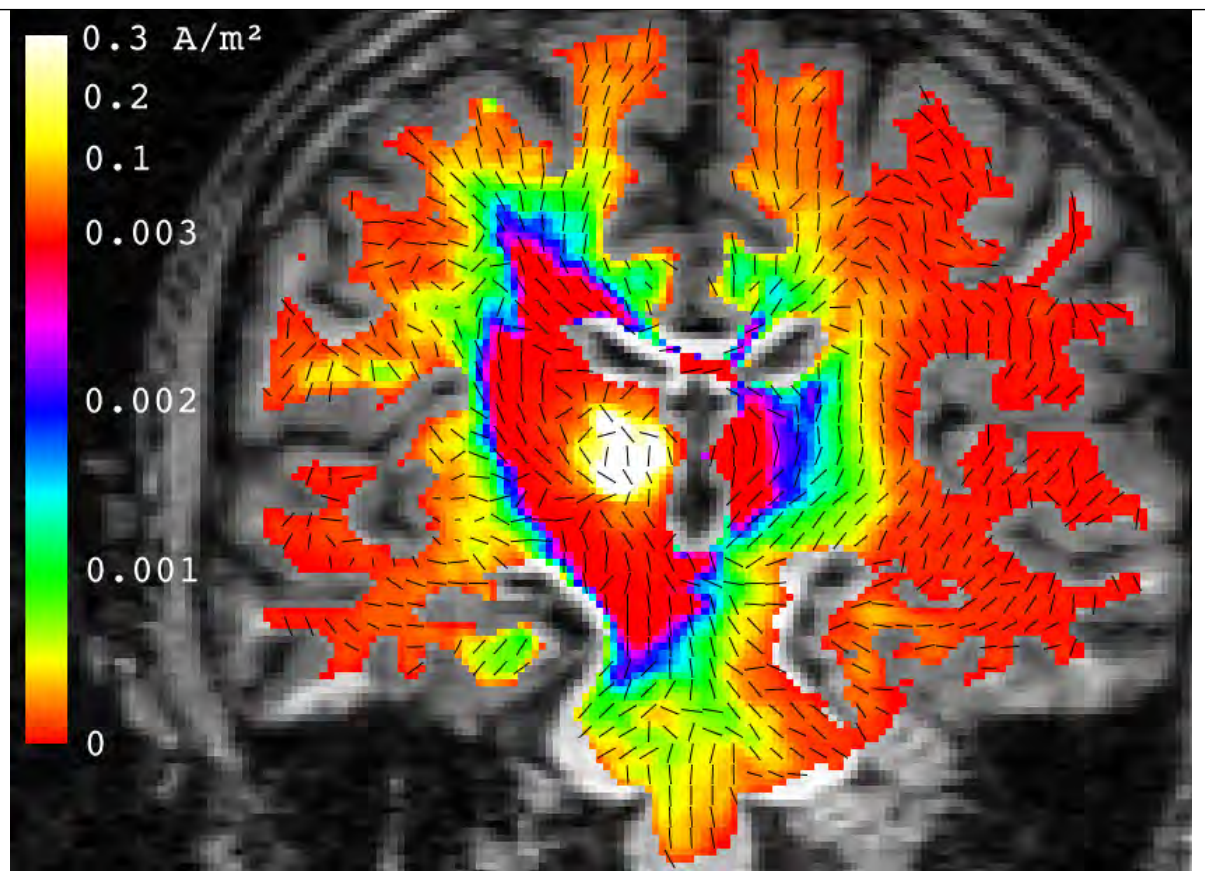

Figure 12. 


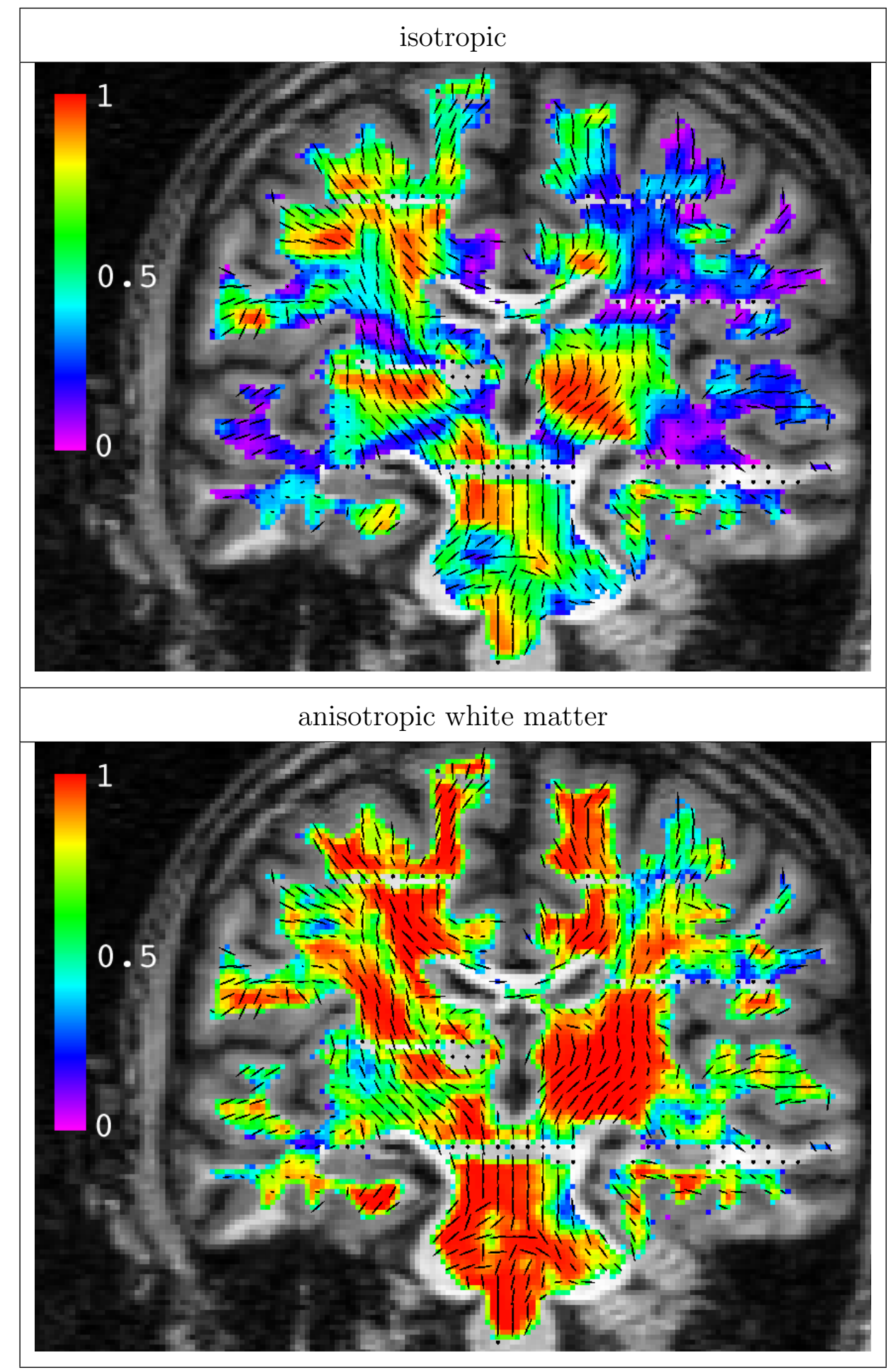

Figure 13. 


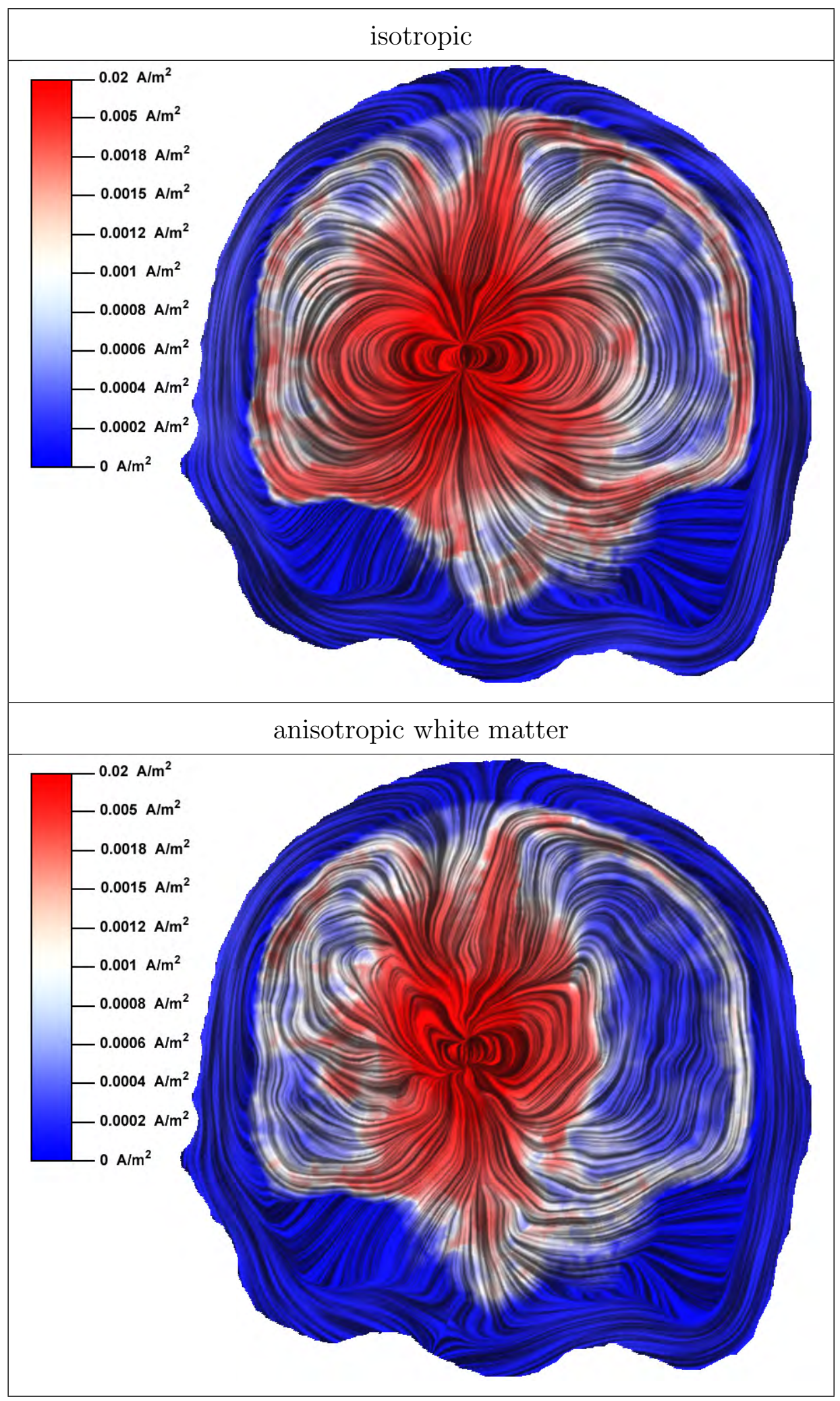

Figure 14. 